\title{
Concise Assembly of the Polycyclic Frameworks Associated with the Hapalindole and Fischerindole Alkaloids
}

\author{
Martin G. Banwell, ${ }^{\star}$ Xinghua Ma, Rebecca M. Taylor and Anthony C. Willis \\ Research School of Chemistry, Australian National University, Canberra, ACT 0200, Australia \\ mgb@rsc.anu.edu.au
}

\section{$\begin{array}{ll}\text { Contents } & \text { Page }\end{array}$}

$\begin{array}{ll}\text { General Experimental Procedures } & \text { S2 }\end{array}$

Experimental Procedures and Product Characterization for Compounds 5-14 and 16-25 S3-S25

X-ray Crystallographic Data for Compounds 8, 13a and 13b $\quad$ S26-S28

$\begin{array}{ll}\text { References } & \text { S29 }\end{array}$

${ }^{1} \mathrm{H}$ or ${ }^{13} \mathrm{C}$ NMR Spectra of Compounds 6-13 and 17-24 S30-S51 


\section{General Experimental Procedures}

Melting points were measured on a Stanford Research Systems Optimelt - Automated Melting Point System and are uncorrected. Proton $\left({ }^{1} \mathrm{H}\right)$ and carbon $\left({ }^{13} \mathrm{C}\right)$ NMR spectra were recorded on either a Varian Gemini $300 \mathrm{MHz}$ or Varian Inova $600 \mathrm{MHz}$ spectrometer. Unless otherwise specified, spectra were acquired at $20{ }^{\circ} \mathrm{C}$ in deuterochloroform $\left(\mathrm{CDCl}_{3}\right)$ that had been filtered through basic alumina immediately prior to use. Chemical shifts are recorded as $\delta$ values in parts per million $(\mathrm{ppm})$. Infrared spectra $\left(v_{\max }\right)$ were recorded on a Perkin-Elmer 1800 Series FTIR Spectrometer and samples were analyzed as $\mathrm{KBr}$ disks (for solids) or as thin films on $\mathrm{KBr}$ plates (for oils). Low-resolution mass spectra were recorded on a Micromass-Waters LC-ZMD single quadrupole liquid chromatograph-MS or a VG Quattro II triple quadrupole MS instrument using electron impact techniques. High-resolution mass spectra were recorded on an AUTOSPEC instrument. Flash chromatographic separations were carried out using the protocols defined by Still et al. ${ }^{1}$ Dichloromethane (DCM) was distilled from calcium hydride and THF was distilled, under nitrogen, from sodium benzophenone ketyl. Where necessary, reactions were performed under a nitrogen or argon atmosphere. 


\section{Experimental Procedures and Product Characterization for Compounds: 5-14 and 16-25}

\section{6,6-Dibromobicyclo[3.1.0]hexane (5)}
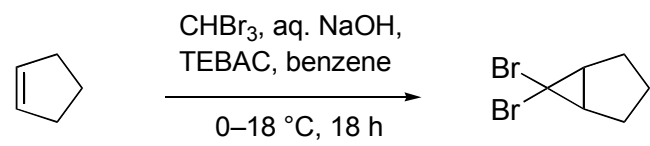

\section{Method A}

5

Sodium hydroxide ( $25 \mathrm{~mL}$ of a $50 \% \mathrm{w} / \mathrm{v}$ aqueous solution) was added to a vigorously stirred mixture of cyclopentene $(4.85 \mathrm{~g}, 71.2 \mathrm{mmol})$, benzyltriethylammonium chloride $(300 \mathrm{mg}, 1.32$ $\mathrm{mmol})$ and bromoform $(10 \mathrm{~mL}, 114.5 \mathrm{mmol})$ in benzene $(15 \mathrm{~mL})$ that was maintained at $0{ }^{\circ} \mathrm{C}$ (icebath). After addition was complete the reaction mixture was placed under a nitrogen atmosphere, allowed to warm to room temperature overnight, then partitioned between hexane $(100 \mathrm{~mL})$ and brine $(100 \mathrm{~mL})$. The separated aqueous layer was extracted with ethyl acetate $(3 \times 25 \mathrm{~mL})$ and the combined organic phases were washed with brine $(2 \times 150 \mathrm{~mL})$ before being dried $\left(\mathrm{MgSO}_{4}\right)$, filtered and concentrated under reduced pressure. The ensuing brown oil was subjected to flash chromatography (silica, hexane elution) and concentration of the appropriate fractions $\left(R_{\mathrm{f}} 0.6\right.$ in 1:20 v/v ethyl acetate/hexane) afforded the title compound $\mathbf{5}^{2}(15.72 \mathrm{~g}, 92 \%)$ as a clear, colorless oil, bp $54-56{ }^{\circ} \mathrm{C} / 4 \mathrm{mmHg}$ (lit. ${ }^{2}$ bp $63{ }^{\circ} \mathrm{C} / 2.9 \mathrm{mmHg}$ ).

${ }^{1} \mathbf{H}$ NMR $\left(300 \mathrm{MHz}, \mathrm{CDCl}_{3}\right) \delta 2.23$ (m, $2 \mathrm{H}$ ), 2.11-1.97 (complex m, $2 \mathrm{H}$ ), 1.93-1.81 (complex m, $2 \mathrm{H}), 1.78-1.64$ (complex m, $2 \mathrm{H}$ ).

${ }^{13}$ C NMR $\left(75 \mathrm{MHz}, \mathrm{CDCl}_{3}\right) \delta 40.8(\mathrm{C}), 39.9(\mathrm{CH}), 29.5\left(\mathrm{CH}_{2}\right), 25.9\left(\mathrm{CH}_{2}\right)$.

IR $(\mathrm{KBr}) v_{\max } 2930,2860,1467,1441,1318,1285,1074,1005,969,890,746 \mathrm{~cm}^{-1}$.

EI MS $m / z(70 \mathrm{eV})$ 242, 240 and $238\left(\mathrm{M}^{+*}, 3,6\right.$ and 3\%), 201, 199 and 197 (5, 10 and 6), 161 and 159 (36 and 35), 79 (100), 57 (42).

HRMS Found: $\mathrm{M}^{+\bullet}, 237.8982 . \mathrm{C}_{6} \mathrm{H}_{8}{ }^{79} \mathrm{Br}_{2}$ requires $\mathrm{M}^{+\bullet}, 237.8993$;

Found: $\mathrm{M}^{+\bullet}, 241.8954 . \mathrm{C}_{6} \mathrm{H}_{8}{ }^{81} \mathrm{Br}_{2}$ requires $\mathrm{M}^{+\bullet}, 241.8952$.

\section{Method B}

A flask containing a mixture of powdered sodium hydroxide (16.50 g, $412.5 \mathrm{mmol}$ ), benzyltriethylammonium chloride (300 mg, $1.32 \mathrm{mmol})$, cyclopentene (4.93 g, $72.3 \mathrm{mmol})$ and 
bromoform $(10 \mathrm{~mL}, 137.4 \mathrm{mmol})$ in DCM (40 mL) was fitted with a reflux condenser and subjected to sonication. After $1 \mathrm{~h}$ TLC analysis indicated that the reaction was complete so the cooled mixture was filtered through a short pad of Celite ${ }^{\mathrm{TM}}$ that was then washed with DCM (100 $\mathrm{mL})$. The combined filtrates were concentrated under reduced pressure and the brown oil so obtained was subjected to flash chromatography (silica, hexane elution). Concentration of the appropriate fractions $\left(R_{\mathrm{f}} 0.6\right.$ in 1:20 v/v ethyl acetate/hexane) afforded compound 5 (15.45 g, 89\%) as a clear, colorless oil that was identical, in all respects, with the material prepared via Method A.

\section{3-(2-Bromo-2-cyclohexen-1-yl)indole (6)}

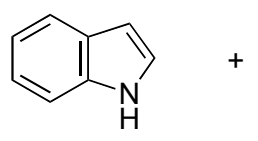

3

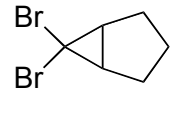

5

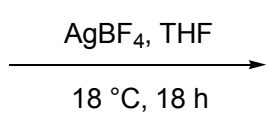

$18^{\circ} \mathrm{C}, 18 \mathrm{~h}$

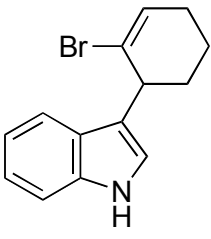

6

Silver tetrafluoroborate $(399 \mathrm{mg}, 2.05 \mathrm{mmol}$ ) was added, in one portion, to a magnetically stirred solution of cyclopropane 5 (246 mg, $1.02 \mathrm{mmol})$ and indole (3) $(120 \mathrm{mg}, 1.02 \mathrm{mmol})$ in anhydrous THF (15 mL). The resulting solution was protected from light and then stirred at ambient temperature for $18 \mathrm{~h}$. After this time the reaction mixture was filtered through a plug of Celite ${ }^{\mathrm{TM}}$ that was washed with ethyl acetate $(200 \mathrm{~mL})$ and the combined filtrates were concentrated under reduced pressure to provide a viscous and brown oil. Subjection of this material to flash chromatography (silica, 1:20 v/v ethyl acetate/hexane elution) and concentration of the appropriate fractions $\left(R_{\mathrm{f}} 0.3\right.$ in 1:5:11 v/v/v ethyl acetate/DCM/hexane) afforded the title compound 6 (218 $\mathrm{mg}$, $77 \%$ ) as a white solid, $\mathrm{mp} 77-80{ }^{\circ} \mathrm{C}$.

${ }^{1}$ H NMR $\left(300 \mathrm{MHz}, \mathrm{CDCl}_{3}\right) \delta 7.97$ (broad s, $\left.1 \mathrm{H}\right), 7.66(\mathrm{~d}, J 7.8 \mathrm{~Hz}, 1 \mathrm{H}), 7.40$ (dd, $J 7.8$ and 1.2 Hz, 1 H), 7.28-7.16 (complex m, 2 H), 7.04 (d, J 2.0 Hz, 1 H), 6.36 (m, 1 H), 4.06 (m, 1 H), 2.272.20 (complex m, 2 H), 2.16-2.09 (complex m, 2 H), 1.70-1.59 (complex m, 2 H).

${ }^{13}$ C NMR (75 MHz, $\left.\mathrm{CDCl}_{3}\right) \delta 136.6(\mathrm{C}), 131.0(\mathrm{CH}), 126.6(\mathrm{C}), 124.9(\mathrm{C}), 123.0(\mathrm{CH}), 122.1$ $(\mathrm{CH}), 119.5(\mathrm{CH}), 118.9(\mathrm{CH}), 117.7(\mathrm{C}), 111.4(\mathrm{CH}), 41.4(\mathrm{CH}), 31.5\left(\mathrm{CH}_{2}\right), 27.9\left(\mathrm{CH}_{2}\right), 17.9$ $\left(\mathrm{CH}_{2}\right)$. 
IR $(\mathrm{KBr}) \boldsymbol{v}_{\max } 3420,3055,2934,2858,1644,1456,1419,1223,1094,983,742 \mathrm{~cm}^{-1}$.

EI MS $m / z(70 \mathrm{eV}) 277$ and $275\left(\mathrm{M}^{+\bullet}, 94\right.$ and 93\%), 249 and 247 (35 and 33), 248 and 246 (36 and 38), 196 and 194 (75 and 51), 168 (88), 167 (86), 117 (100), 84 (88), 77 (39).

HRMS Found: $\mathrm{M}^{+\bullet}, 275.0311 . \mathrm{C}_{14} \mathrm{H}_{14}{ }^{79} \mathrm{BrN}$ requires $\mathrm{M}^{+\bullet}, 275.0310$;

Found: $\mathrm{M}^{+\bullet}, 277.0301 . \mathrm{C}_{14} \mathrm{H}_{14}{ }^{81} \mathrm{BrN}$ requires $\mathrm{M}^{+\bullet}, 277.0289$.

\section{1-Methyl-3-(2-bromo-2-cyclohexen-1-yl)indole (7)}

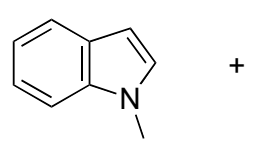

4

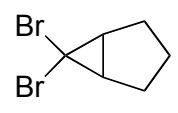

5

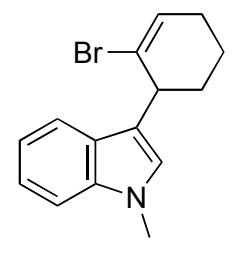

7

Cyclopropane 5 (1.50 g, $6.27 \mathrm{mmol})$ was subjected to reaction with silver tetrafluoroborate $(2.43 \mathrm{~g}$, $12.48 \mathrm{mmol}$ ) in the presence of 1-methylindole (4) $(825 \mathrm{mg}, 6.23 \mathrm{mmol})$ under the same conditions as employed for the conversion $3+5 \rightarrow \mathbf{6}$ described immediately above. The brown oil obtained on workup was subjected to flash chromatography (silica, 1:20 v/v ethyl acetate/hexane elution) and concentration of the relevant fractions $\left(R_{\mathrm{f}} \quad 0.5\right.$ in $1: 5: 11 \mathrm{v} / \mathrm{v} / \mathrm{v}$ ethyl acetate/DCM/hexane) afforded the title compound 7 (1.22 g, 67\%) as a clear, colorless oil.

${ }^{1}$ H NMR $\left(300 \mathrm{MHz}, \mathrm{CDCl}_{3}\right) \delta 7.70(\mathrm{~d}, J 6.8 \mathrm{~Hz}, 1 \mathrm{H}), 7.39(\mathrm{~d}, J 6.8 \mathrm{~Hz}, 1 \mathrm{H}), 7.33$ (t, J 6.8 Hz, 1 H), 7.22 (t, J $6.8 \mathrm{~Hz}, 1 \mathrm{H}), 6.97(\mathrm{~s}, 1 \mathrm{H}), 6.40$ (t, J $3.9 \mathrm{~Hz}, 1 \mathrm{H}), 4.08($ broad s, $1 \mathrm{H}), 3.82(\mathrm{~s}, 3 \mathrm{H})$, 2.33-2.24 (complex m, 2 H), 2.22-2.11 (complex m, 2 H), 1.76-1.63 (complex m, 2 H).

${ }^{13} \mathrm{C}$ NMR (75 MHz, $\left.\mathrm{CDCl}_{3}\right) \delta 137.4(\mathrm{C}), 130.8(\mathrm{CH}), 127.6(\mathrm{CH}), 127.0(\mathrm{C}), 125.0(\mathrm{C}), 121.6$ $(\mathrm{CH}), 118.9(\mathrm{CH}), 118.8(\mathrm{CH}), 116.1(\mathrm{C}), 109.4(\mathrm{CH}), 41.3(\mathrm{CH}), 32.8\left(\mathrm{CH}_{3}\right), 31.7\left(\mathrm{CH}_{2}\right), 27.9$ $\left(\mathrm{CH}_{2}\right), 17.9\left(\mathrm{CH}_{2}\right)$.

IR $(\mathrm{KBr}) v_{\max } 3051,2935,2875,1641,1614,1473,1374,1329,1233,1012,982,739 \mathrm{~cm}^{-1}$.

EI MS $m / z(70 \mathrm{eV}) 291$ and $289\left(\mathrm{M}^{+\bullet}, 79\right.$ and 77\%), 263 and 261 (31 and 26), 262 and 260 (37 and 35), 210 and 208 (55 and 35), 182 (70), 181 (76), 131 (100), 91 (42).

HRMS Found: $\mathrm{M}^{+\bullet}, 289.0471 . \mathrm{C}_{15} \mathrm{H}_{16}{ }^{79} \mathrm{BrN}$ requires $\mathrm{M}^{+\bullet}, 289.0466$;

Found: $\mathrm{M}^{+\bullet}, 291.0448 . \mathrm{C}_{15} \mathrm{H}_{16}{ }^{81} \mathrm{BrN}$ requires $\mathrm{M}^{+\bullet}, 291.0446$. 


\section{Methyl 6-(1'-Methylindol-3'-yl)cyclohex-1-ene Carboxylate (8)}

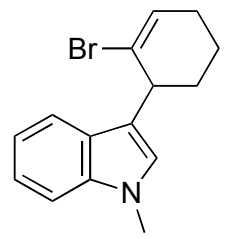

7

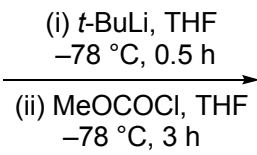

$-78^{\circ} \mathrm{C}, 3 \mathrm{~h}$

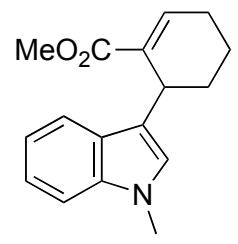

8

$t$-Butyllithium $(1.7 \mathrm{~mL}$ of a $1.7 \mathrm{M}$ solution in pentane, $3.06 \mathrm{mmol})$ was added, dropwise, to a magnetically stirred solution of bromocyclohexene 7 (407 mg, $1.40 \mathrm{mmol}$ ) in anhydrous THF (20 $\mathrm{mL}$ ) maintained at $-78{ }^{\circ} \mathrm{C}$ (dry-ice/acetone bath) under a nitrogen atmosphere. The resulting solution was stirred at $-78{ }^{\circ} \mathrm{C}$ for $0.5 \mathrm{~h}$ before being treated with solution of methyl chloroformate $(160 \mu \mathrm{L}, 2.07 \mathrm{mmol})$ in THF $(5 \mathrm{~mL})$. The ensuing mixture was stirred at $-78{ }^{\circ} \mathrm{C}$ for a further $3 \mathrm{~h}$ then treated with $\mathrm{NH}_{4} \mathrm{Cl}$ (50 $\mathrm{mL}$ of a saturated aqueous solution). The separated aqueous phase was extracted with ethyl acetate $(3 \times 15 \mathrm{~mL})$ and the combined organic phases were then dried $\left(\mathrm{MgSO}_{4}\right)$, filtered and concentrated under reduced pressure. Subjection of the ensuing residue to flash chromatography (silica, 1:25 v/v ethyl acetate/hexane elution) and concentration of the appropriate fractions $\left(R_{\mathrm{f}} 0.5\right.$ in 2:5:11 v/v/v ethyl acetate/DCM/hexane) afforded the title ester 8 (215 mg, 57\%) as a white, crystalline solid, mp 96-99 ${ }^{\circ} \mathrm{C}$.

${ }^{1}$ H NMR $\left(300 \mathrm{MHz}, \mathrm{CDCl}_{3}\right) \delta 7.72(\mathrm{~d}, J 7.8 \mathrm{~Hz}, 1 \mathrm{H}), 7.35-7.10$ (complex m, $\left.4 \mathrm{H}\right), 6.65$ (s, $\left.1 \mathrm{H}\right)$, 4.35 (broad s, 1 H), 3.74 (s, 3 H), 3.65 (s, 3 H), 2.41-2.22 (complex m, 2 H), 2.20-2.01 (complex m, $1 \mathrm{H}), 1.91-1.80$ (complex m, $1 \mathrm{H}), 1.60-1.51$ (complex m, $2 \mathrm{H}$ ).

${ }^{13} \mathrm{C}$ NMR (75 MHz, $\left.\mathrm{CDCl}_{3}\right) \delta 168.2(\mathrm{C}), 140.5(\mathrm{CH}), 137.6(\mathrm{C}), 132.6(\mathrm{C}), 127.2(\mathrm{CH}), 127.0(\mathrm{C})$, $121.5(\mathrm{CH}), 119.2(\mathrm{CH}), 118.7(\mathrm{CH}), 118.2(\mathrm{C}), 109.3(\mathrm{CH}), 51.7\left(\mathrm{CH}_{3}\right), 32.7\left(\mathrm{CH}\right.$ or $\left.\mathrm{CH}_{3}\right), 30.5$ $\left(\mathrm{CH}\right.$ or $\left.\mathrm{CH}_{3}\right), 28.8\left(\mathrm{CH}_{2}\right), 25.9\left(\mathrm{CH}_{2}\right), 17.1\left(\mathrm{CH}_{2}\right)$.

IR $(\mathrm{KBr}) v_{\max } 2936,1714,1644,1472,1434,1328,1257,1241,1093,1068,738 \mathrm{~cm}^{-1}$.

EI MS m/z (70 eV) 269 (M+•, 100\%), 254 (20), 241 (48), 240 (70), 238 (37), 210 (65), 209 (90), 208 (88), 182 (70), 181 (78), 167 (56), 131 (95), 91 (46), 77 (33).

HRMS Found: $\mathrm{M}^{+\bullet}, 269.1417 . \mathrm{C}_{17} \mathrm{H}_{19} \mathrm{NO}_{2}$ requires: $\mathrm{M}^{+\bullet}, 269.1416$. 


\section{1-[6-(1-Methylindol-3-yl)cyclohex-1-enyl]ethanone (9)}

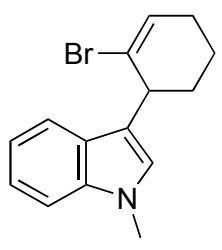

7

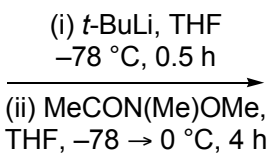

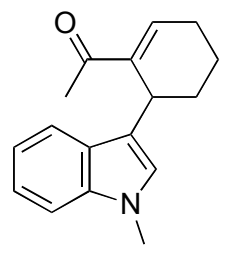

9

$t$-Butyllithium $(1.2 \mathrm{~mL}$ of a $1.7 \mathrm{M}$ solution in pentane, $2.04 \mathrm{mmol})$ was added, dropwise, to a magnetically stirred solution of bromocyclohexene 7 (262 mg, $0.90 \mathrm{mmol}$ ) in anhydrous THF (18 $\mathrm{mL}$ ) maintained at $-78{ }^{\circ} \mathrm{C}$ (dry-ice/acetone bath) under a nitrogen atmosphere. The resulting solution was stirred at $-78{ }^{\circ} \mathrm{C}$ for $0.5 \mathrm{~h}$ then treated with a solution of $N$-methoxy- $N$ methylacetamide $^{3}(140 \mathrm{mg}, 1.36 \mathrm{mmol})$ in THF $(2 \mathrm{~mL})$. The ensuing mixture was allowed to warm to $0{ }^{\circ} \mathrm{C}$ over $4 \mathrm{~h}$ then $\mathrm{NH}_{4} \mathrm{Cl}$ (50 mL of a saturated aqueous solution) was added. The separated aqueous phase was extracted with ethyl acetate $(3 \times 15 \mathrm{~mL})$ and the combined organic phases were then dried $\left(\mathrm{MgSO}_{4}\right)$, filtered and concentrated under reduced pressure. Subjection of the ensuing residue to flash chromatography (silica, 1:10 v/v ethyl acetate/hexane elution) and concentration of the appropriate fractions $\left(R_{\mathrm{f}} 0.3\right.$ in 2:5:11 v/v/v ethyl acetate/DCM/hexane) afforded compound 9 (135 mg, 59\%) as a white, crystalline solid, $\mathrm{mp} 124-126^{\circ} \mathrm{C}$.

${ }^{1} \mathbf{H}$ NMR $\left(300 \mathrm{MHz}, \mathrm{CDCl}_{3}\right) \delta 7.70$ (d, J 7.8 Hz, $\left.1 \mathrm{H}\right), 7.35-7.18$ (complex m, $2 \mathrm{H}$ ), 7.16-7.08 (complex m, $2 \mathrm{H}), 6.56$ (s, $1 \mathrm{H}), 4.37$ (broad s, $1 \mathrm{H}), 3.71$ (s, $3 \mathrm{H}), 2.45-2.22$ (complex m, $2 \mathrm{H})$, 2.25 (s, $3 \mathrm{H}$ ), 2.06-2.01 (complex m, 1 H), 1.87-1.76 (complex m, 1 H), 1.60-1.51 (complex m, $2 \mathrm{H})$.

${ }^{13}$ C NMR (75 MHz, $\left.\mathrm{CDCl}_{3}\right) \delta 199.3(\mathrm{C}), 141.3(\mathrm{C}), 140.2(\mathrm{CH}), 137.6(\mathrm{C}), 127.3(\mathrm{CH}), 126.9(\mathrm{C})$, $121.7(\mathrm{CH}), 119.2(\mathrm{CH}), 118.8(\mathrm{CH}), 118.1(\mathrm{C}), 109.3(\mathrm{CH}), 32.7\left(\mathrm{CH}_{3}\right), 30.0(\mathrm{CH}), 28.9\left(\mathrm{CH}_{2}\right)$, $26.2\left(\mathrm{CH}_{3}\right), 26.1\left(\mathrm{CH}_{2}\right), 17.3\left(\mathrm{CH}_{2}\right)$.

IR $(\mathrm{KBr}) v_{\max } 3051,2933,1667,1636,1616,1472,1423,1328,1231,1154,1013,903,739 \mathrm{~cm}^{-1}$.

EI MS $m / z(70 \mathrm{eV}) 253\left(\mathrm{M}^{+\bullet}, 99 \%\right), 238$ (30), 224 (38), 210 (45), 182 (50), 167 (29), 132 (30), 131 (100), 77 (16), 43 (27).

HRMS Found: $\mathrm{M}^{+\bullet}, 253.1464 . \mathrm{C}_{17} \mathrm{H}_{19} \mathrm{NO}$ requires $\mathrm{M}^{+\bullet}, 253.1467$. 
(1RS,2RS)-Methyl 2-(1-Methylindol-3-yl)cyclohexene Carboxylate (10a) and (1RS,2SR)Methyl 2-(1-Methylindol-3-yl)cyclohexene Carboxylate (10b)

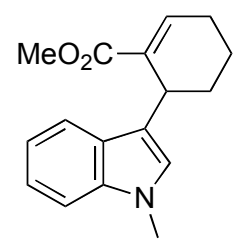

8

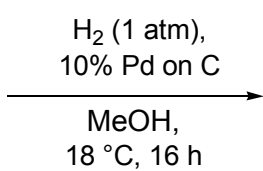

$18^{\circ} \mathrm{C}, 16 \mathrm{~h}$

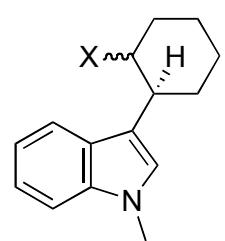

$10 \mathrm{a} X=\beta-\mathrm{CO}_{2} \mathrm{Me}$

$10 \mathrm{~b} X=\alpha-\mathrm{CO}_{2} \mathrm{Me}$

$10 \%$ Palladium on carbon $(31 \mathrm{mg})$ was added to a magnetically stirred solution of compound 8 (62 $\mathrm{mg}, 0.23 \mathrm{mmol})$ in methanol $(1.5 \mathrm{~mL})$ and the ensuing mixture was stirred at $18{ }^{\circ} \mathrm{C}$ under an atmosphere of hydrogen for $16 \mathrm{~h}$ then filtered through a plug of Celite ${ }^{\mathrm{TM}}$ that was washed with methanol $(30 \mathrm{~mL})$. Concentration of the combined filtrates under reduced pressure provided a light-yellow oil that was subjected to flash chromatography (silica, gradient elution using 1:50 to 1:25 v/v ethyl acetate/hexane). In this manner two fractions, A and B, were obtained.

Concentration of fraction A $\left(R_{\mathrm{f}} 0.3\right.$ in 1:5:11 v/v/v ethyl acetate/DCM/hexane $)$ afforded a white crystalline solid (32 mg, 51\%), mp $64-66^{\circ} \mathrm{C}$, tentatively identified as ester 10a.

${ }^{1} \mathbf{H}$ NMR $\left(300 \mathrm{MHz}, \mathrm{CDCl}_{3}\right) \delta 7.65(\mathrm{~d}, J 7.8 \mathrm{~Hz}, 1 \mathrm{H}), 7.27-7.18(\mathrm{~m}, 2 \mathrm{H}), 7.10(\mathrm{t}, J 7.8 \mathrm{~Hz}, 1 \mathrm{H})$, $6.86(\mathrm{~s}, 1 \mathrm{H}), 3.72(\mathrm{~s}, 3 \mathrm{H}), 3.39(\mathrm{~s}, 3 \mathrm{H}), 3.15$ (m, $1 \mathrm{H}), 2.71$ (m, $1 \mathrm{H}), 2.12-2.01$ (complex m, 2 H), 1.90-1.82 (complex m, $2 \mathrm{H}), 1.67$ (m, $1 \mathrm{H})$, 1.59-1.31 (complex m, $3 \mathrm{H}$ ).

${ }^{13}$ C NMR (75 MHz, $\left.\mathrm{CDCl}_{3}\right) \delta 176.4(\mathrm{C}), 137.1(\mathrm{C}), 127.2(\mathrm{C}), 125.3(\mathrm{CH}), 121.4(\mathrm{CH}), 119.6$ $(\mathrm{CH}), 118.7(\mathrm{C}), 118.6(\mathrm{CH}), 109.2(\mathrm{CH}), 51.4\left(\mathrm{CH}_{3}\right), 50.6(\mathrm{CH}), 37.3(\mathrm{CH}), 34.4\left(\mathrm{CH}_{2}\right), 32.8$ $\left(\mathrm{CH}_{3}\right), 30.6\left(\mathrm{CH}_{2}\right), 26.5\left(\mathrm{CH}_{2}\right), 25.6\left(\mathrm{CH}_{2}\right)$.

IR $(\mathrm{KBr}) v_{\max } 3050,2931,2855,1734,1471,1447,1374,1249,1165,1037,1013,738 \mathrm{~cm}^{-1}$.

EI MS $m / z(70 \mathrm{eV}) 272$ [(M+H) $\left.)^{+}, 55 \%\right], 271\left(\mathrm{M}^{+\bullet}, 98\right), 240$ (15), 228 (23), 212 (38), 184 (39), 171 (55), 170 (100), 157 (63), 144 (77), 131 (44), 115 (30), 42 (27).

HRMS Found: $\mathrm{M}^{+\bullet}, 271.1569 . \mathrm{C}_{17} \mathrm{H}_{21} \mathrm{NO}_{2}$ requires: $\mathrm{M}^{+\bullet}, 271.1572$. 
Concentration of fraction B $\left(R_{\mathrm{f}} 0.4\right.$ in 1:5:11 v/v/v ethyl acetate/DCM/hexane) afforded a white solid (30 mg, 49\%), mp 106-108 ${ }^{\circ} \mathrm{C}$, tentatively identified as ester $\mathbf{1 0 b}$.

${ }^{1} \mathbf{H}$ NMR $\left(300 \mathrm{MHz}, \mathrm{CDCl}_{3}\right) \delta 7.58(\mathrm{~d}, J 7.8 \mathrm{~Hz}, 1 \mathrm{H}), 7.28(\mathrm{~d}, J 7.8 \mathrm{~Hz}, 1 \mathrm{H}), 7.21(\mathrm{t}, J 7.8 \mathrm{~Hz}, 1$ H), 7.09 (t, J $7.8 \mathrm{~Hz}, 1 \mathrm{H}), 6.96(\mathrm{~s}, 1 \mathrm{H}), 3.74(\mathrm{~s}, 3 \mathrm{H}), 3.50(\mathrm{~m}, 1 \mathrm{H}), 3.41(\mathrm{~s}, 3 \mathrm{H}), 3.02(\mathrm{~m}, 1 \mathrm{H})$, 2.31 (m, 1 H), 2.02 (m, 1 H), 1.90-1.70 (complex m, 4 H), 1.55-1.42 (complex m, 2 H).

${ }^{13}$ C NMR (75 MHz, $\left.\mathrm{CDCl}_{3}\right) \delta 175.2(\mathrm{C}), 136.6(\mathrm{C}), 127.8(\mathrm{C}), 126.6(\mathrm{CH}), 121.4(\mathrm{CH}), 118.9$ $(\mathrm{CH}), 118.6(\mathrm{CH}), 116.8(\mathrm{C}), 109.2(\mathrm{CH}), 51.1\left(\mathrm{CH}_{3}\right), 45.4(\mathrm{CH}), 35.0(\mathrm{CH}), 32.8\left(\mathrm{CH}_{3}\right), 29.4$ $\left(\mathrm{CH}_{2}\right), 27.3\left(\mathrm{CH}_{2}\right), 24.5\left(\mathrm{CH}_{2}\right), 23.2\left(\mathrm{CH}_{2}\right)$.

IR (KBr) $v_{\max } 3047,2930,2855,1732,1466,1447,1376,1326,1251,1162,1033,738 \mathrm{~cm}^{-1}$.

EI MS m/z (70 eV) 272 [(M+H) $\left.)^{+}, 75 \%\right], 271\left(\mathrm{M}^{+\bullet}, 100\right), 240$ (23), 212 (47), 184 (48), 171 (72), 170 (98), 157 (82), 144 (94), 132 (63), 131 (72), 115 (41), 42 (37).

HRMS Found: $\mathrm{M}^{+\bullet}, 271.1576 . \mathrm{C}_{17} \mathrm{H}_{21} \mathrm{NO}_{2}$ requires: $\mathrm{M}^{+\bullet}, 271.1572$.

$(1 R S, 2 S R)-1-[2-(1 '-M e t h y l i n d o l-3 '-y l) c y c l o h e x y l] e t h a n o n e \quad(11 a)$ and $(1 R S, 2 R S)-1-[2-(1 '-$ Methylindol-3'-yl)cyclohexyl]ethanone (11b)

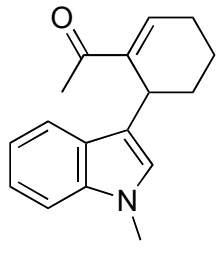

9

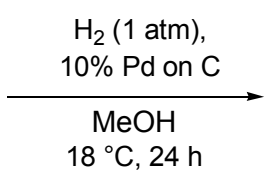

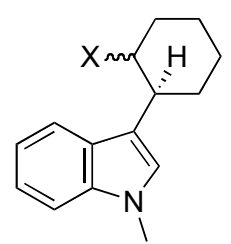

11a $X=\beta-\mathrm{COMe}$ $11 \mathrm{~b} X=\alpha-\mathrm{COMe}$

A solution of enone 9 (130 mg, $0.54 \mathrm{mmol})$ in methanol (2 mL) was maintained, for $24 \mathrm{~h}$, under atmosphere of hydrogen and in the presence of $10 \%$ palladium on carbon $(65 \mathrm{mg})$ using the same conditions as employed for the conversion $8 \rightarrow \mathbf{1 0 a}+\mathbf{1 0 b}$ described immediately above. The yellow oil obtained on work-up was subjected to flash chromatography (silica, gradient elution using 1:25 to $1: 15 \mathrm{v} / \mathrm{v}$ ethyl acetate/hexane). In this manner two fractions, A and B, were obtained.

Concentration of fraction A $\left(R_{\mathrm{f}} 0.4\right.$ in 2:5:11 v/v/v ethyl acetate/DCM/hexane) afforded ketone $11 \boldsymbol{a}$ (25 mg, 19\%) as a clear, colorless oil. 
${ }^{1}$ H NMR $\left(300 \mathrm{MHz}, \mathrm{CDCl}_{3}\right) \delta 7.64(\mathrm{~d}, J 7.8 \mathrm{~Hz}, 1 \mathrm{H}), 7.31-7.20$ (complex m, $\left.2 \mathrm{H}\right), 7.13$ (m, $\left.1 \mathrm{H}\right)$, $6.91(\mathrm{~s}, 1 \mathrm{H}), 3.73(\mathrm{~s}, 3 \mathrm{H}), 3.70$ (complex m, $1 \mathrm{H}), 3.06(\mathrm{~m}, 1 \mathrm{H}), 2.25$ (complex m, $1 \mathrm{H}), 1.88$ (s, $3 \mathrm{H}), 2.05-1.78$ (complex m, $4 \mathrm{H}$ ), 1.62-1.53 (complex m, $1 \mathrm{H}$ ), 1.51-1.40 (complex m, $2 \mathrm{H}$ ).

${ }^{13}$ C NMR (75 MHz, $\left.\mathrm{CDCl}_{3}\right) \delta 212.0(\mathrm{C}), 136.6(\mathrm{C}), 127.8(\mathrm{C}), 127.1(\mathrm{CH}), 121.6(\mathrm{CH}), 118.8$ $(\mathrm{CH}), 118.7(\mathrm{CH}), 115.7(\mathrm{C}), 109.3(\mathrm{CH}), 52.7(\mathrm{CH}), 34.5\left(\mathrm{CH}_{3}\right), 32.8(\mathrm{CH}), 30.4\left(\mathrm{CH}_{2}\right), 29.6$ $\left(\mathrm{CH}_{3}\right), 25.6\left(\mathrm{CH}_{2}\right), 23.8\left(\mathrm{CH}_{2}\right), 23.4\left(\mathrm{CH}_{2}\right)$.

IR $(\mathrm{KBr}) \boldsymbol{v}_{\max } 3052,2929,2853,1704,1471,1447,1375,1350,1330,1247,1160,739 \mathrm{~cm}^{-1}$.

EI MS m/z (70 eV) $256\left[(\mathrm{M}+\mathrm{H})^{+}, 86 \%\right], 255\left(\mathrm{M}^{+\bullet}, 98\right), 240$ (7), 212 (97), 197 (62), 184 (56), 182 (57), 171 (82), 170 (98), 157 (98), 144 (100), 131 (82), 115 (66), 77 (38), 43 (72), 42 (63).

HRMS Found: $\mathrm{M}^{+\bullet}, 255.1632 . \mathrm{C}_{17} \mathrm{H}_{21} \mathrm{NO}$ requires $\mathrm{M}^{+\bullet}, 255.1623$.

Concentration of fraction B $\left(R_{\mathrm{f}} 0.5\right.$ in 2:5:11 v/v/v ethyl acetate/DCM/hexane $)$ afforded ketone $\mathbf{1 1 b}$ (83 $\mathrm{mg}, 63 \%)$ as a clear, colorless oil.

${ }^{1} \mathbf{H}$ NMR $\left(300 \mathrm{MHz}, \mathrm{CDCl}_{3}\right) \delta 7.69(\mathrm{~d}, J 7.8 \mathrm{~Hz}, 1 \mathrm{H}), 7.29-7.19$ (complex m, $\left.2 \mathrm{H}\right), 7.11(\mathrm{t}, J$ 7.8 Hz, $1 \mathrm{H}), 6.81$ (s, $1 \mathrm{H}), 3.72$ (s, $3 \mathrm{H}), 3.08$ (m, $1 \mathrm{H}), 2.91$ (complex m, $1 \mathrm{H}), 2.07$ (m, $1 \mathrm{H}), 1.97-$ 1.83 (complex m, $3 \mathrm{H}$ ), 1.83 (s, $3 \mathrm{H}$ ), 1.65-1.40 (complex m, $4 \mathrm{H}$ ).

${ }^{13}$ C NMR (75 MHz, $\left.\mathrm{CDCl}_{3}\right) \delta 213.4(\mathrm{C}), 137.2(\mathrm{C}), 126.9(\mathrm{C}), 125.8(\mathrm{CH}), 121.6(\mathrm{CH}), 119.5$ $(\mathrm{CH}), 118.8(\mathrm{CH}), 118.2(\mathrm{C}), 109.4(\mathrm{CH}), 57.6(\mathrm{CH}), 37.7\left(\mathrm{CH}_{3}\right), 34.8\left(\mathrm{CH}_{2}\right), 32.8(\mathrm{CH}), 30.1$ $\left(\mathrm{CH}_{2}\right), 28.7\left(\mathrm{CH}_{3}\right), 26.6\left(\mathrm{CH}_{2}\right), 25.8\left(\mathrm{CH}_{2}\right)$.

IR $(\mathrm{KBr}) \boldsymbol{v}_{\max } 2928,2853,1705,1472,1357,1249,1156,738 \mathrm{~cm}^{-1}$.

EI MS $m / z(70 \mathrm{eV}) 256\left[(\mathrm{M}+\mathrm{H})^{+}, 30 \%\right], 255\left(\mathrm{M}^{+\bullet}, 91\right), 212$ (62), 170 (100), 157 (50), $144(75)$, 131 (30), 115 (23), 77 (12), 69 (47), 43 (44).

HRMS Found: $\mathrm{M}^{+\bullet}, 255.1622 . \mathrm{C}_{17} \mathrm{H}_{21} \mathrm{NO}$ requires $\mathrm{M}^{+\bullet}, 255.1623$.

(1'RS,2'SR)-1-Methyl 3-[2'-(Propen-2'-yl)cyclohexan-1'-yl]indole (12a)

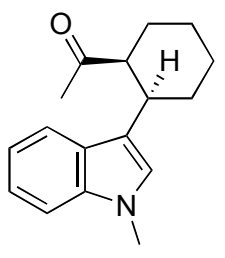

$11 \mathrm{a}$

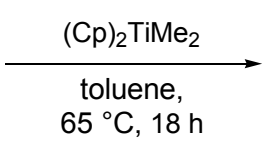

$65^{\circ} \mathrm{C}, 18 \mathrm{~h}$

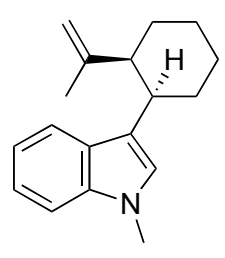

$12 a$ 
Dimethyltitanocene $(1.7 \mathrm{~mL}$ of a $0.5 \mathrm{M}$ solution in toluene, $0.85 \mathrm{mmol}$ ) was added to a solution of ketone 11a (72 $\mathrm{mg}, 0.28 \mathrm{mmol})$ in toluene $(12 \mathrm{~mL})$. The resulting mixture was protected from light and heated at $65^{\circ} \mathrm{C}$ under a nitrogen atmosphere for $18 \mathrm{~h}$. After this time, TLC analysis indicated that no starting material remained so the cooled reaction mixture was diluted with hexane $(25 \mathrm{~mL})$. The ensuing yellow-orange precipitate was removed by filtration and the combined filtrates were concentrated under reduced pressure to give a bright-yellow oil. Subjection of this material to flash chromatography (silica, 1:100 v/v ethyl acetate/hexane elution) and concentration of the appropriate fractions $\left(R_{\mathrm{f}} 0.4\right.$ in 1:20 v/v ethyl acetate/hexane) afforded the title alkene $12 \mathrm{a}(58 \mathrm{mg}$, $82 \%)$ as a clear, light-yellow oil.

${ }^{1}$ H NMR $\left(300 \mathrm{MHz}, \mathrm{CDCl}_{3}\right) \delta 7.64(\mathrm{~d}, J 6.8 \mathrm{~Hz}, 1 \mathrm{H}), 7.31(\mathrm{~d}, J 6.8 \mathrm{~Hz}, 1 \mathrm{H}), 7.23$ (t, J $6.8 \mathrm{~Hz}, 1$ H), 7.12 (t, J $6.8 \mathrm{~Hz}, 1 \mathrm{H}), 7.02$ (s, $1 \mathrm{H}), 4.72$ (m, $2 \mathrm{H}), 3.80(\mathrm{~m}, 1 \mathrm{H}), 3.76(\mathrm{~s}, 3 \mathrm{H}), 2.48$ (complex m, $1 \mathrm{H}), 2.05$ (m, $1 \mathrm{H}), 1.96-1.80$ (complex m, $4 \mathrm{H}), 1.62$ (s, $3 \mathrm{H}), 1.51-1.41$ (complex m, $3 \mathrm{H})$. ${ }^{13}$ C NMR (75 MHz, $\left.\mathrm{CDCl}_{3}\right) \delta 149.2(\mathrm{C}), 136.3(\mathrm{C}), 128.7(\mathrm{C}), 127.2(\mathrm{CH}), 121.2(\mathrm{CH}), 119.0$ $(\mathrm{CH}), 118.4(\mathrm{CH}), 115.4(\mathrm{C}), 109.5\left(\mathrm{CH}_{2}\right), 109.0(\mathrm{CH}), 46.8(\mathrm{CH}), 33.6\left(\mathrm{CH}_{3}\right), 32.8(0)(\mathrm{CH})$, 32.7(6) $\left(\mathrm{CH}_{2}\right), 27.0\left(\mathrm{CH}_{2}\right), 26.4\left(\mathrm{CH}_{2}\right), 22.7\left(\mathrm{CH}_{3}\right), 21.9\left(\mathrm{CH}_{2}\right)$.

IR $(\mathrm{KBr}) v_{\max } 3053,2929,2852,1640,1483,1463,1448,1374,1310,1247,1154,1114,885,737$ $\mathrm{cm}^{-1}$.

EI MS $m / z(70 \mathrm{eV}) 254$ [(M+H) $\left.{ }^{+}, 60 \%\right], 253\left(\mathrm{M}^{+\bullet}, 96\right), 238$ (32), $210(25), 197$ (46), $196(56), 182$ (45), 171 (70), 170 (100), 157 (78), 144 (97), 131 (50), 115 (53), 77 (30), 42 (42).

HRMS Found: $\mathrm{M}^{+\bullet}, 253.1823 . \mathrm{C}_{18} \mathrm{H}_{23} \mathrm{~N}$ requires $\mathrm{M}^{+\bullet}, 253.1830$.

\section{$(1 ' R S, 2 ' R S)-1-M e t h y l$ 3-[2'-(Propen-2'-yl)cyclohexan-1'-yl]indole (12b)}

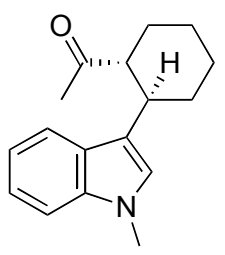

11b

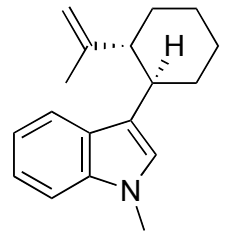

$12 b$ 
Reaction of ketone $11 b(76 \mathrm{mg}, 0.30 \mathrm{mmol})$ with dimethyltitanocene in the same manner as described immediately above for the conversion 11a $\rightarrow$ 12a afforded a light-yellow oil on workup. Subjection of this material to flash chromatography (silica, 1:100 v/v ethyl acetate/hexane elution) and concentration of the appropriate fractions $\left(R_{\mathrm{f}} 0.4\right.$ in 1:20 v/v ethyl acetate) afforded the title alkene $\mathbf{1 2 b}$ (60 $\mathrm{mg}, 80 \%)$ as a clear, pale-yellow oil.

${ }^{1}$ H NMR $\left(300 \mathrm{MHz}, \mathrm{CDCl}_{3}\right) \delta 7.64(\mathrm{~d}, J 7.8 \mathrm{~Hz}, 1 \mathrm{H}), 7.28-7.17$ (complex m, $\left.2 \mathrm{H}\right), 7.09$ (t, $J 7.8$ Hz, 1 H), 6.79 (broad s, 1 H), 4.66 (broad s, 1 H), 4.54 (broad s, 1 H), 3.72 (broad s, 3 H), 2.85 (t, $J 12.3 \mathrm{~Hz}, 1 \mathrm{H}), 2.53(\mathrm{t}, J 12.3 \mathrm{~Hz}, 1 \mathrm{H}), 2.10(\mathrm{~m}, 1 \mathrm{H}), 1.86(\mathrm{~m}, 3 \mathrm{H}), 1.55(\mathrm{~s}, 3 \mathrm{H}), 1.55-1.47$ (complex m, $4 \mathrm{H}$ ).

${ }^{13}$ C NMR (75 MHz, $\left.\mathrm{CDCl}_{3}\right) \delta 149.9(\mathrm{C}), 136.9$ (C), 127.5 (C), $125.7(\mathrm{CH}), 121.1(\mathrm{CH}), 119.5(1)$ (C), 119.4(8) (C), $118.3\left(\mathrm{CH}_{2}\right), 110.6(\mathrm{CH}), 109.2(\mathrm{CH}), 51.4(\mathrm{CH}), 38.8\left(\mathrm{CH}_{3}\right), 36.3\left(\mathrm{CH}_{2}\right), 33.8$ $\left(\mathrm{CH}_{2}\right), 32.7(\mathrm{CH}), 27.2\left(\mathrm{CH}_{2}\right), 26.8\left(\mathrm{CH}_{2}\right), 19.5\left(\mathrm{CH}_{3}\right)$.

IR $(\mathrm{KBr}) v_{\max } 3056,2923,2851,1643,1471,1446,1373,1325,1249,1014,883,798,736 \mathrm{~cm}^{-1}$.

EI MS m/z (70 eV) $254\left[(\mathrm{M}+\mathrm{H})^{+}, 91 \%\right], 253\left(\mathrm{M}^{+\bullet}, 100\right), 238$ (47), 210 (44), 197 (71), 196 (85), 182 (74), 171 (93), 170 (99), 157 (96), 144 (97), 131 (74), 115 (75), 77 (51), 42 (68).

HRMS Found: $\mathrm{M}^{+\bullet}, 253.1832 . \mathrm{C}_{18} \mathrm{H}_{23} \mathrm{~N}$ requires: $\mathrm{M}^{+\bullet}, 253.1830$.

\section{(6cRS,10aSR)-1,2,6c,7,8,9,10,10a-Octahydro-1,1,2-trimethylindeno[2,1-b]indole (13a)}

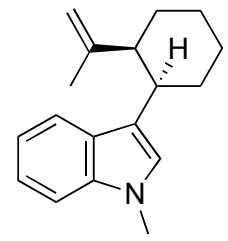

$12 a$

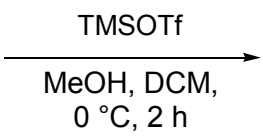

$0{ }^{\circ} \mathrm{C}, 2 \mathrm{~h}$

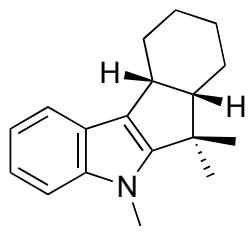

$13 a$

A magnetically stirred solution of compound 12a $(57 \mathrm{mg}, 0.22 \mathrm{mmol})$ in anhydrous DCM (10 $\mathrm{mL})$ maintained at $0{ }^{\circ} \mathrm{C}$ (ice bath) under a nitrogen atmosphere was treated with trimethylsilyl methanesulfonate $(160 \mu \mathrm{L}, 0.88 \mathrm{mmol})$ then methanol $(18 \mu \mathrm{L}, 0.44 \mathrm{mmol})$. The ensuing mixture was maintained at $0{ }^{\circ} \mathrm{C}$ for a further $2 \mathrm{~h}$ then treated with $\mathrm{NaHCO}_{3}(25 \mathrm{~mL}$ of a saturated aqueous solution). The separated aqueous phase was extracted with DCM $(2 \times 10 \mathrm{~mL})$ and the combined filtrates were dried $\left(\mathrm{MgSO}_{4}\right)$, filtered and concentrated under reduced pressure to provide a pink 
oil. Subjection of this material to flash chromatography (silica, 1:100 v/v ethyl acetate/hexane elution) and concentration of the appropriate fractions $\left(R_{\mathrm{f}} 0.5\right.$ in 1:20 v/v ethyl acetate/hexane) afforded the title compound $13 \boldsymbol{a}(48 \mathrm{mg}, 84 \%)$ as a white, crystalline solid, mp $77-79{ }^{\circ} \mathrm{C}$.

${ }^{1}$ H NMR $\left(300 \mathrm{MHz}, \mathrm{CDCl}_{3}\right) \delta 7.64(\mathrm{~d}, J 7.6 \mathrm{~Hz}, 1 \mathrm{H}), 7.27$ (d, J 7.6 Hz, $\left.1 \mathrm{H}\right), 7.18-7.06$ (complex m, $2 \mathrm{H}), 3.76(\mathrm{~s}, 3 \mathrm{H}), 3.32(\mathrm{~m}, 1 \mathrm{H}), 2.46(\mathrm{~m}, 1 \mathrm{H}), 2.04(\mathrm{~m}, 1 \mathrm{H}), 1.76-1.61$ (complex m, $3 \mathrm{H})$, 1.60-1.45 (complex m, $4 \mathrm{H}), 1.40$ (s, $3 \mathrm{H}), 1.39$ (s, $3 \mathrm{H}$ ).

${ }^{13}$ C NMR (75 MHz, $\mathrm{CDCl}_{3}$ ) $\delta 151.0(\mathrm{C}), 141.1$ (C), 124.2 (C), $119.9(\mathrm{CH}), 119.8$ (C), $118.9(\mathrm{CH})$, $118.6(\mathrm{CH}), 109.3(\mathrm{CH}), 53.8(\mathrm{CH}), 43.2(\mathrm{C}), 37.2(\mathrm{CH}), 30.3\left(\mathrm{CH}_{3}\right), 30.1\left(\mathrm{CH}_{2}\right), 27.0\left(\mathrm{CH}_{3}\right), 24.4$ $\left(\mathrm{CH}_{2}\right), 24.3\left(\mathrm{CH}_{3}\right), 24.2\left(\mathrm{CH}_{2}\right), 23.9\left(\mathrm{CH}_{2}\right)$.

IR $(\mathrm{KBr}) v_{\max } 3051,2925,2853,1465,1377,1362,1233,1132,1013,916,738 \mathrm{~cm}^{-1}$.

EI MS m/z (70 eV) 254 [(M+H) $\left.)^{+}, 39 \%\right], 253\left(\mathrm{M}^{+\bullet}, 92\right), 238$ (100), 210 (75), 194 (39), 182 (38), 167 (26), 157 (22), 131 (17), 97 (26), 57 (28), 43 (28).

HRMS Found: $\mathrm{M}^{+\bullet}, 253.1831 . \mathrm{C}_{18} \mathrm{H}_{23} \mathrm{~N}$ requires $\mathrm{M}^{+\bullet}, 253.1830$.

\section{$(6 \mathrm{c} R S, 10 \mathrm{a} R S)-1,2,6 \mathrm{c}, 7,8,9,10,10 \mathrm{a}-O c t a h y d r o-1,1,2-t r i m e t h y l i n d e n o[2,1-b]$ indole (13b)}

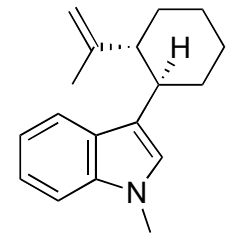

$12 b$

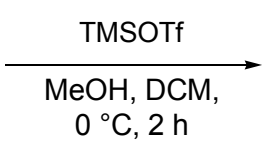

$0{ }^{\circ} \mathrm{C}, 2 \mathrm{~h}$

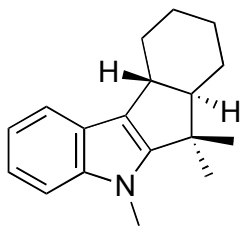

$13 b$

Cyclization of alkene 12b (47 $\mathrm{mg}, 0.18 \mathrm{mmol}$ ) in the same manner as described immediately above for the conversion 12a $\rightarrow$ 13a afforded a light-yellow oil on workup. Subjection of this material to flash chromatography (silica, 1:100 v/v ethyl acetate/hexane elution) and concentration of the appropriate fractions $\left(R_{\mathrm{f}} 0.4\right.$ in 1:20 v/v ethyl acetate/hexane) afforded the title alkene $\mathbf{1 3 b}$ $(38 \mathrm{mg}, 81 \%)$ as a white crystalline solid, $\mathrm{mp} 132-134^{\circ} \mathrm{C}$.

${ }^{1}$ H NMR $\left(300 \mathrm{MHz}, \mathrm{CDCl}_{3}\right) \delta 7.54$ (d, J 7.6 Hz, $\left.1 \mathrm{H}\right), 7.29$ (d, J 8.0 Hz, $\left.1 \mathrm{H}\right), 7.18-7.06$ (complex m, 2 H), 3.78 (s, 3 H), 2.70 (m, 1 H), 2.61 (m, 1 H), 1.98-1.87 (complex m, 3 H), 1.78 (m, 1 H), 1.59-1.40 (complex m, $4 \mathrm{H}), 1.49$ (s, $3 \mathrm{H}), 1.11$ (s, $3 \mathrm{H})$. 


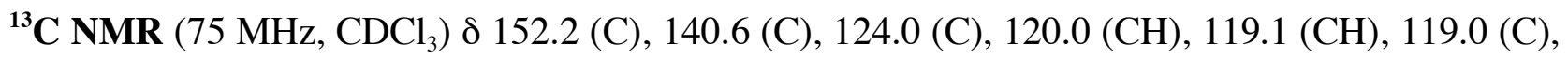
$118.7(\mathrm{CH}), 109.4(\mathrm{CH}), 64.2(\mathrm{CH}), 43.1(\mathrm{CH}), 41.6(\mathrm{C}), 31.4\left(\mathrm{CH}_{2}\right), 30.4\left(\mathrm{CH}_{3}\right), 27.0\left(\mathrm{CH}_{2}\right), 26.7$ $\left(\mathrm{CH}_{2}\right), 25.7\left(\mathrm{CH}_{3}\right), 25.0\left(\mathrm{CH}_{2}\right), 20.1\left(\mathrm{CH}_{3}\right)$.

IR $(\mathrm{KBr}) v_{\max } 2916,2846,1464,1381,1346,1230,1132,738,721 \mathrm{~cm}^{-1}$.

EI MS $m / z(70 \mathrm{eV}) 254$ [(M+H) $\left.{ }^{+}, 60 \%\right], 253\left(\mathrm{M}^{+\bullet}, 98 \%\right), 238(100), 210(60), 196(41), 194$ (52), 182 (39), 167 (32), 144 (27), 131 (21), 97 (44), 69 (45), 57 (56), 43 (57).

HRMS Found: $\mathrm{M}^{+\bullet}, 253.1828 . \mathrm{C}_{18} \mathrm{H}_{23} \mathrm{~N}$ requires $\mathrm{M}^{+\bullet}, 253.1830$.

\section{1,6-Heptadien-4-one (14)}

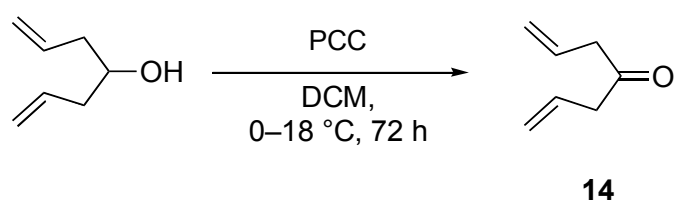

Pyridinium chlorochromate $(20.0 \mathrm{~g}, 92.8 \mathrm{mmol})$ was added, in two portions over $1 \mathrm{~h}$, to a magnetically stirred solution of 1,6-heptadiene-4-ol $(5.0 \mathrm{~g}, 44.6 \mathrm{mmol})$ in anhydrous DCM (200 $\mathrm{mL}$ ) maintained at $0{ }^{\circ} \mathrm{C}$ (ice-bath) under a nitrogen atmosphere. Upon completion of the addition the resulting mixture was protected from light and allowed to warm to ambient temperature. Stirring was continued for a further $72 \mathrm{~h}$ then the reaction mixture was filtered through a plug of silica gel that was washed with diethyl ether $(800 \mathrm{~mL})$. The combined filtrates were concentrated under reduced pressure and residue subjected to flash chromatography (silica, 1:10 v/v diethyl ether/DCM elution). Concentration of the appropriate fractions $\left(R_{\mathrm{f}} 0.4\right.$ in $1: 5: 11 \mathrm{v} / \mathrm{v} / \mathrm{v}$ ethyl acetate/DCM/hexane) afforded ketone $14^{4}(4.3 \mathrm{~g}, 90 \%)$ as a clear, colorless oil.

${ }^{1}$ H NMR (300 MHz, $\mathrm{CDCl}_{3}$ ) $\delta$ 5.97-5.83 (complex m, $2 \mathrm{H}$ ), 5.20-5.09 (complex m, $4 \mathrm{H}$ ), 3.20 (d, $J 7.1 \mathrm{~Hz}, 4 \mathrm{H})$.

${ }^{13} \mathrm{C}$ NMR $\left(75 \mathrm{MHz}, \mathrm{CDCl}_{3}\right) \delta 206.6(\mathrm{C}), 130.4(\mathrm{CH}), 119.1\left(\mathrm{CH}_{2}\right), 47.2\left(\mathrm{CH}_{2}\right)$.

IR $(\mathrm{KBr}) v_{\max } 3082,2956,2926,2856,1719,1640,1425,1395,1330,1138,1049,993,919 \mathrm{~cm}^{-1}$.

EI MS m/z (70 eV) $110\left(\mathrm{M}^{+\bullet}, 25 \%\right), 97$ (74), 85 (85), 83 (75), 71 (95), 69 (80), 57 (100), 43 (96), 41 (78).

HRMS Found: $\mathrm{M}^{+\bullet}, 110.0732 . \mathrm{C}_{7} \mathrm{H}_{10} \mathrm{O}$ requires $\mathrm{M}^{+\bullet}, 110.0732$. 


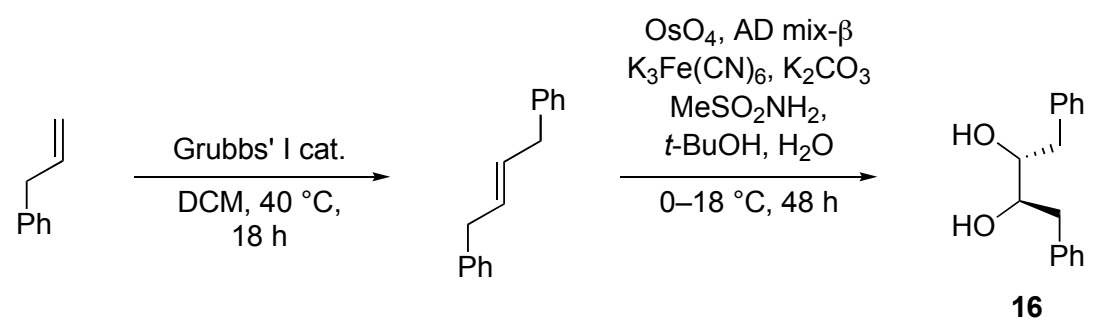

Step (i): Grubbs' first-generation catalyst (1.4 g, $1.75 \mathrm{mmol})$ was added to a deoxygenated and magnetically stirred solution of allyl benzene (4.0 g, $33.9 \mathrm{mmol})$ in anhydrous DCM (80 $\mathrm{mL})$ maintained under a nitrogen atmosphere. The resulting solution was heated at reflux for $18 \mathrm{~h}$ then cooled and concentrated under reduced pressure. The residue so-obtained was subjected to flash chromatography (silica, 1:50 v/v ethyl acetate/hexane elution) to provide, after concentration of the appropriate fractions $\left(R_{\mathrm{f}} 0.5\right.$ in 1:40 v/v ethyl acetate/hexane), $E$-1,4-diphenyl-2-butene ${ }^{5}$ (3.1 g, $89 \%$ ) as a clear, colorless oil. This material slowly crystallized on standing to give a white solid, mp 39-40 ${ }^{\circ} \mathrm{C}$ (lit. $\left.{ }^{5} \mathrm{mp} 40.5-42^{\circ} \mathrm{C}\right)$.

${ }^{1}$ H NMR (300 MHz, $\mathrm{CDCl}_{3}$ ) $\delta$ 7.41-7.33 (complex m, $4 \mathrm{H}$ ), 7.29-7.24 (complex m, $\left.6 \mathrm{H}\right)$, 5.775.72 (complex m, $2 \mathrm{H}), 3.44(\mathrm{~m}, 4 \mathrm{H})$.

${ }^{13}$ C NMR $\left(75 \mathrm{MHz}, \mathrm{CDCl}_{3}\right) \delta 140.9(\mathrm{C}), 130.6(\mathrm{CH}), 128.7(\mathrm{CH}), 128.5(\mathrm{CH}), 126.1(\mathrm{CH}), 39.1$ $\left(\mathrm{CH}_{2}\right)$.

IR $(\mathrm{KBr}) v_{\max } 3027,2903,2839,1602,1494,1452,1074,1029,969,739,697 \mathrm{~cm}^{-1}$.

EI MS $m / z(70 \mathrm{eV}) 208\left(\mathrm{M}^{+\bullet}, 94 \%\right), 194$ (34), 130 (71), 129 (55), 118 (80), 117 (100), 116 (65), 115 (92), 104 (86), 91 (99), 77 (57), 65 (63), 51 (55).

HRMS Found: $\mathrm{M}^{+\bullet}, 208.1248 . \mathrm{C}_{16} \mathrm{H}_{16}$ requires: $\mathrm{M}^{+\bullet}, 208.1252$.

Step (ii): Potassium ferricyanide (14.0 g, $48.25 \mathrm{mmol})$, potassium carbonate (6.2 g, $44.86 \mathrm{mmol})$, AD-mix- $\beta(150 \mathrm{mg}, 0.19 \mathrm{mmol})$ and osmium tetroxide $(1.6 \mathrm{~mL}$ of a $0.1 \mathrm{M}$ solution in $t$-butanol, $0.16 \mathrm{mmol})$ were dissolved in $t$-butanol/water $(100 \mathrm{~mL}$ of a $1: 1 \mathrm{v} / \mathrm{v}$ mixture $)$ and the resulting solution treated with methanesulfonamide $(1.4 \mathrm{~g}, 14.72 \mathrm{mmol})$ then cooled to $0{ }^{\circ} \mathrm{C}$. $E-1,4-$ Diphenyl-2-butene $(3.1 \mathrm{~g}, 14.88 \mathrm{mmol})$ was then added, in one portion, and the ensuing mixture allowed to warm to room temperature. Stirring was continued for $48 \mathrm{~h}$ and the reaction mixture was then treated with sodium thiosulfate $(24.0 \mathrm{~g}, 96.7 \mathrm{mmol})$ at $0{ }^{\circ} \mathrm{C}$ and stirring continued for a 
further $1 \mathrm{~h}$. The separated aqueous phase was extracted with ethyl acetate $(3 \times 500 \mathrm{~mL})$ and the combined organic fractions were washed with brine $(2 \times 250 \mathrm{~mL})$ before being dried $\left(\mathrm{MgSO}_{4}\right)$, filtered and concentrated under reduced pressure. Subjection of the ensuing material to flash chromatography (silica, 1:2 v/v ethyl acetate/hexane elution) and concentration of the relevant fractions $\left(R_{\mathrm{f}} 0.3\right.$ in 8:5:11 v/v/v ethyl acetate/DCM/hexane) afforded diol $\mathbf{1 6}^{6}(3.2 \mathrm{~g}, 89 \%)$ as a white, crystalline solid, mp $125-127^{\circ} \mathrm{C}\left(\right.$ lit. $\left.^{6} \mathrm{mp} 126-128^{\circ} \mathrm{C}\right)$.

${ }^{1}$ H NMR (300 MHz, $\mathrm{CDCl}_{3}$ ) $\delta$ 7.36-7.23 (complex m, $10 \mathrm{H}$ ), 3.78 (m, $2 \mathrm{H}$ ), 2.98-2.83 (complex m, $4 \mathrm{H}), 2.03$ (s, $2 \mathrm{H})$.

${ }^{13}$ C NMR $\left(75 \mathrm{MHz}, \mathrm{CDCl}_{3}\right) \delta 138.2(\mathrm{C}), 129.5(\mathrm{CH}), 128.8(\mathrm{CH}), 126.7(\mathrm{CH}), 74.1(\mathrm{CH}), 40.5$ $\left(\mathrm{CH}_{2}\right)$.

IR $(\mathrm{KBr}) v_{\max } 3329,3026,2910,1602,1495,1453,1101,1040,944,749,698,501 \mathrm{~cm}^{-1}$.

EI MS m/z (70 eV) $242\left(\mathrm{M}^{+\bullet}, 4 \%\right), 150$ (63), 133 (68), 121 (61), 103 (48), 92 (95), 91 (100), 78 (26), 77 (31), 65 (28).

HRMS Found: $\mathrm{M}^{+\bullet}, 242.1308 . \mathrm{C}_{16} \mathrm{H}_{18} \mathrm{O}_{2}$ requires: $\mathrm{M}^{+\bullet}, 242.1307$.

Specific rotation $[\alpha]_{\mathrm{D}}-2.0\left(c 0.5, \mathrm{CHCl}_{3}\right)$.

(4R,5R)-2,2-Diallyl-4,5-dimethyl-[1,3]dioxolane (17)

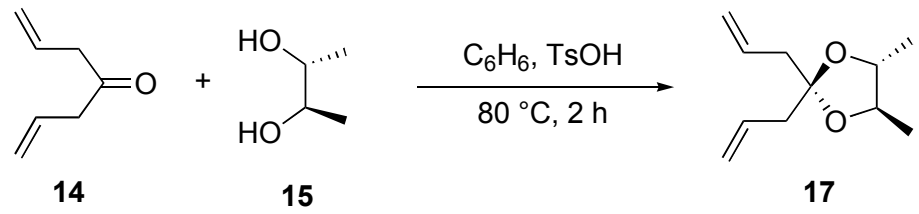

p-Toluenesulfonic acid ( $7 \mathrm{mg}, 0.04 \mathrm{mmol}$ ) was added to a magnetically stirred solution of ketone 14 (729 $\mathrm{mg}, 6.62 \mathrm{mmol})$ and 2R,3R-butanediol (15) $(954 \mathrm{mg}, 10.6 \mathrm{mmol})$ in benzene $(25 \mathrm{~mL})$ contained in a flask fitted with a Dean-Stark trap, dry-ice condenser and calcium chloride drying tube. The resulting mixture was heated at reflux for $2 \mathrm{~h}$ then cooled and treated with sodium hydroxide ( $25 \mathrm{~mL}$ of a $1.0 \mathrm{M}$ aqueous solution). The separated organic phase was washed with brine $(2 \times 25 \mathrm{~mL})$ before being dried $\left(\mathrm{MgSO}_{4}\right)$, filtered and concentrated under reduced pressure. Subjection of the ensuing residue to flash chromatography (silica, 1:100 v/v ethyl acetate/hexane 
elution) and concentration of the relevant fractions $\left(R_{\mathrm{f}} \quad 0.4\right.$ in $1: 5: 11 \mathrm{v} / \mathrm{v} / \mathrm{v}$ ethyl acetate/DCM/hexane) afforded ketal $17(880 \mathrm{mg}, 73 \%)$ as a pale-yellow oil.

${ }^{1}$ H NMR (300 MHz, CDCl $)$ ) 5.90-5.76 (complex m, 2 H), 5.10 (m, 2 H), 5.06 (m, 2 H), 3.60 (m, $2 \mathrm{H}), 2.38$ (s, $2 \mathrm{H}), 2.36$ (s, $2 \mathrm{H}), 1.22(\mathrm{~s}, 3 \mathrm{H}), 1.21$ (d, J $3.9 \mathrm{~Hz}, 6 \mathrm{H})$.

${ }^{13}$ C NMR $\left(75 \mathrm{MHz}, \mathrm{CDCl}_{3}\right) \delta 133.3(\mathrm{CH}), 118.3\left(\mathrm{CH}_{2}\right), 109.5(\mathrm{C}), 78.9(\mathrm{CH}), 43.5\left(\mathrm{CH}_{2}\right), 16.7$ $(\mathrm{CH})$.

IR $(\mathrm{KBr}) v_{\max } 3078,2979,2878,1642,1432,1377,1316,1293,1155,1097,999,980,914,842$ $\mathrm{cm}^{-1}$.

EI MS $m / z(70 \mathrm{eV}) 182\left(\mathrm{M}^{+\bullet}, 5 \%\right), 167$ (15), 141 (83), 69 (100), 55 (29), 41 (66).

HRMS Found: $\mathrm{M}^{+\bullet}, 182.1307 . \mathrm{C}_{11} \mathrm{H}_{18} \mathrm{O}_{2}$ requires: $\mathrm{M}^{+\bullet}, 182.1307$.

Specific rotation $[\alpha]_{\mathrm{D}}-21.6\left(c 1.2, \mathrm{CHCl}_{3}\right)$.

\section{(2R,3R)-2,2-Diallyl-4,5-dibenzyl-[1,3]dioxolane (18)}

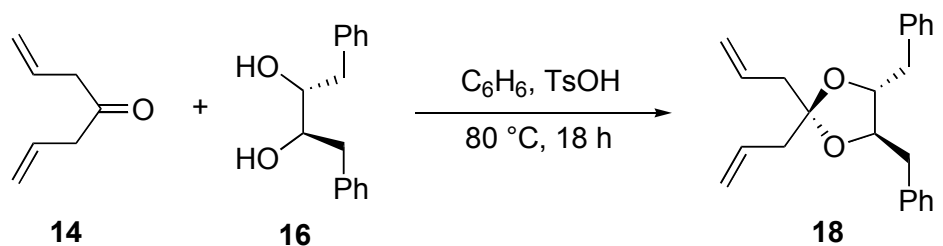

p-Toluenesulfonic acid ( $8 \mathrm{mg}, 0.04 \mathrm{mmol}$ ) was added to a magnetically stirred solution of ketone $14(240 \mathrm{mg}, 2.18 \mathrm{mmol})$ and diol $16(235 \mathrm{mg}, 0.97 \mathrm{mmol})$ in benzene (40 mL) contained in a flask fitted with a Dean-Stark trap, dry-ice condenser and calcium chloride drying tube. The resulting mixture was heated at reflux for 18 hours then cooled and treated with sodium hydroxide $(50 \mathrm{~mL}$ of a 1.0 M aqueous solution). The separated organic phase was washed with brine $(2 \times 30 \mathrm{~mL})$ before being dried $\left(\mathrm{MgSO}_{4}\right)$, filtered and concentrated under reduced pressure. Subjection of the ensuing residue to flash chromatography (silica, 1:100 v/v ethyl acetate/hexane elution) and concentration of the relevant fractions $\left(R_{\mathrm{f}} 0.3\right.$ in 1:5:11 v/v/v ethyl acetate/DCM/hexane $)$ afforded ketal 18 (234 $\mathrm{mg}, 72 \%)$ as a pale-yellow oil.

${ }^{1} \mathbf{H}$ NMR $\left(300 \mathrm{MHz}, \mathrm{CDCl}_{3}\right.$ ) $\delta$ 7.36-7.24 (complex m, $6 \mathrm{H}$ ), 7.23-7.15 (complex m, $4 \mathrm{H}$ ), 5.925.78 (complex m, $2 \mathrm{H}), 5.15$ (m, $2 \mathrm{H}), 5.11$ (m, $2 \mathrm{H}), 3.97$ (m, $2 \mathrm{H}), 2.85$ (dd, J 13.9 and $6.1 \mathrm{~Hz}$, $2 \mathrm{H}), 2.60$ (dd, $J 13.9$ and $4.3 \mathrm{~Hz}, 2 \mathrm{H}), 2.34(\mathrm{~d}, J 7.1 \mathrm{~Hz}, 4 \mathrm{H})$. 
${ }^{13}$ C NMR (75 MHz, $\left.\mathrm{CDCl}_{3}\right) \delta 137.5(\mathrm{C}), 133.5(\mathrm{CH}), 129.7(\mathrm{CH}), 128.5(\mathrm{CH}), 126.6(\mathrm{CH}), 118.3$ $\left(\mathrm{CH}_{2}\right), 110.2(\mathrm{C}), 81.3(\mathrm{CH}), 43.3\left(\mathrm{CH}_{2}\right), 39.0\left(\mathrm{CH}_{2}\right)$.

IR $(\mathrm{KBr}) v_{\max } 3064,3029,2916,1641,1604,1496,1454,1432,1080,915,755,699 \mathrm{~cm}^{-1}$.

EI MS m/z (70 eV) $334\left(\mathrm{M}^{+\bullet}, 31 \%\right), 294$ (65), 293 (99), 243 (34), 207 (48), 133 (64), 129 (68), 117 (33), 105 (43), 91 (100), 69 (93), 41 (84).

HRMS Found: $\mathrm{M}^{+\bullet}, 334.1939 . \mathrm{C}_{23} \mathrm{H}_{26} \mathrm{O}_{2}$ requires $\mathrm{M}^{+\bullet}, 334.1933$.

Specific rotation $[\alpha]_{\mathrm{D}}+0.8\left(c 0.5, \mathrm{CHCl}_{3}\right)$.

\section{(2R,3R)-2,3-Dimethyl-1,4-dioxaspiro[4.4]non-7-ene (19)}

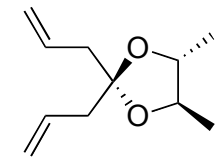

17

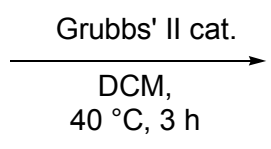

$40^{\circ} \mathrm{C}, 3 \mathrm{~h}$

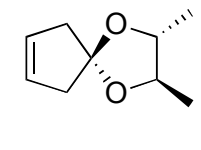

19

Grubbs' second-generation catalyst (40 mg, $0.05 \mathrm{mmol}$ ) was added to a deoxygenated solution of ketal 17 (465 mg, $2.55 \mathrm{mmol})$ in anhydrous DCM (45 mL) and the resulting mixture heated at reflux for $3 \mathrm{~h}$ whilst being maintained under a nitrogen atmosphere. The cooled reaction mixture was concentrated under reduced pressure and the ensuing residue subjected to flash chromatography (silica, 1:100 v/v ethyl acetate/hexane elution). Concentration of the relevant fractions $\left(R_{\mathrm{f}} 0.4\right.$ in 1:5:11 v/v/v ethyl acetate/DCM/hexane) delivered the title compound 19 (299 $\mathrm{mg}, 76 \%$ ) as a clear, colorless oil.

${ }^{1} \mathbf{H}$ NMR $\left(300 \mathrm{MHz}, \mathrm{CDCl}_{3}\right.$ ) $\delta 5.68$ (broad s, $2 \mathrm{H}$ ), 3.63 (complex m, $2 \mathrm{H}$ ), 2.59 (s, $2 \mathrm{H}$ ), 2.58 (s, 2 $\mathrm{H}), 1.26(\mathrm{~d}, J 1.2 \mathrm{~Hz}, 6 \mathrm{H})$.

${ }^{13}$ C NMR $\left(75 \mathrm{MHz}, \mathrm{CDCl}_{3}\right) \delta 128.3(\mathrm{CH}), 116.7(\mathrm{C}), 78.6(\mathrm{CH}), 44.8\left(\mathrm{CH}_{2}\right), 17.2\left(\mathrm{CH}_{3}\right)$.

IR $(\mathrm{KBr}) v_{\max } 3062,2973,2873,1455,1376,1316,1219,1124,1104,953,914,858,660 \mathrm{~cm}^{-1}$.

EI MS $m / z(70 \mathrm{eV}) 154\left(\mathrm{M}^{+\bullet}, 67 \%\right), 82(61), 69(100), 57(85), 55$ (83), 43 (95), 41 (75).

HRMS Found: $\mathrm{M}^{+\bullet}, 154.0997 . \mathrm{C}_{9} \mathrm{H}_{14} \mathrm{O}_{2}$ requires $\mathrm{M}^{+\bullet}, 154.0994$.

Specific rotation $[\alpha]_{\mathrm{D}}-1.2\left(c 0.5, \mathrm{CHCl}_{3}\right)$. 

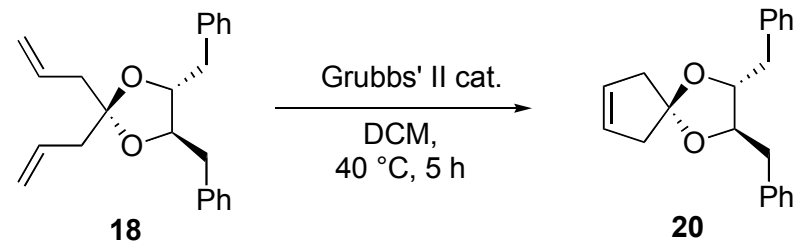

Grubbs' second-generation catalyst $(15 \mathrm{mg}, 0.02 \mathrm{mmol})$ was added to a deoxygenated solution of ketal $18(78 \mathrm{mg}, 0.23 \mathrm{mmol})$ in anhydrous DCM $(15 \mathrm{~mL})$ and the resulting mixture heated at reflux for $5 \mathrm{~h}$ whilst being maintained under a nitrogen atmosphere. The cooled reaction mixture was concentrated under reduced pressure and the ensuing residue subjected to flash chromatography (silica, 1:25 v/v ethyl acetate/hexane elution). Concentration of the relevant fractions $\left(R_{\mathrm{f}} 0.4\right.$ in 1:5:11 v/v/v ethyl acetate/DCM/hexane) afforded the title compound 20 (68 $\mathrm{mg}, 94 \%$ ) as a clear, colorless oil.

${ }^{1} \mathbf{H}$ NMR (300 MHz, $\mathrm{CDCl}_{3}$ ) $\delta$ 7.33-7.21 (complex m, $6 \mathrm{H}$ ), 7.16-7.14 (complex m, $4 \mathrm{H}$ ), 5.69 (broad s, 2 H), 3.98 (m, 2 H), 2.87 (dd, J 13.9 and 5.3 Hz, 2 H), 2.64 (m, 2 H), 2.60 (broad s, 4 H).

${ }^{13}$ C NMR $\left(75 \mathrm{MHz}, \mathrm{CDCl}_{3}\right) \delta 137.6(\mathrm{C}), 129.5(\mathrm{CH}), 128.5(\mathrm{CH}), 128.2(\mathrm{CH}), 126.7(\mathrm{CH}), 117.4$ (C), $81.1(\mathrm{CH}), 44.8\left(\mathrm{CH}_{2}\right), 39.5\left(\mathrm{CH}_{2}\right)$.

IR $(\mathrm{KBr}) \boldsymbol{v}_{\max } 3062,3027,2922,2860,1604,1496,1454,1321,1136,1101,1031,954,746,700$ $\mathrm{cm}^{-1}$.

EI MS m/z (70 eV) $306\left(\mathrm{M}^{+\bullet}, 80 \%\right), 207$ (13), 133 (55), 129 (35), 105 (30), 91 (100), 54 (30).

HRMS Found: $\mathrm{M}^{+\bullet}, 306.1617 . \mathrm{C}_{21} \mathrm{H}_{22} \mathrm{O}_{2}$ requires $\mathrm{M}^{+\bullet}, 306.1620$.

Specific rotation $[\alpha]_{\mathrm{D}}+7.8\left(c 0.5, \mathrm{CHCl}_{3}\right)$.

\section{6,6-Dibromobicyclo[3.1.0]hexan-3-one (2R,3R)-2,3-Dimethylethylene Ketal (21)}

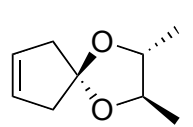

19

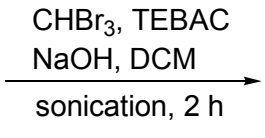

sonication, $2 \mathrm{~h}$

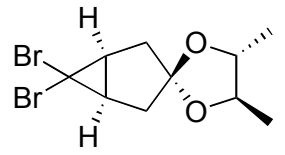

21 
A round-bottomed flask containing a solution of compound 19 (107 mg, $0.69 \mathrm{mmol})$ in DCM (2 $\mathrm{mL}$ ) was charged with benzyltriethylammonium chloride ( $3 \mathrm{mg}, 0.01 \mathrm{mmol})$, bromoform (150 $\mu \mathrm{L}$, $1.72 \mathrm{mmol})$ and powdered sodium hydroxide $(145 \mathrm{mg}, 3.62 \mathrm{mmol})$. The flask was then fitted with a reflux condenser and the resulting mixture subjected to sonication for $2 \mathrm{~h}$. After this time the cooled reaction mixture was filtered through a plug of Celite ${ }^{\mathrm{TM}}$ that was washed with DCM (25 $\mathrm{mL})$. Concentration of the combined filtrates under reduced pressure provided a brown oil that was subjected to flash chromatography (silica, 1:25 v/v ethyl acetate/hexane elution). Concentration of the relevant fractions $\left(R_{\mathrm{f}} 0.4\right.$ in 1:5:11 v/v/v ethyl acetate/DCM/hexane) afforded cyclopropane 21 (205 mg, 91\%) as a white, crystalline solid, mp 56-58 ${ }^{\circ} \mathrm{C}$.

${ }^{1}$ H NMR $\left(300 \mathrm{MHz}, \mathrm{CDCl}_{3}\right.$ ) $\delta 3.53$ (m, 2 H), 2.36-2.19 (complex m, $4 \mathrm{H}$ ), 1.88-1.75 (complex m, $2 \mathrm{H}), 1.26$ (d, J $5.1 \mathrm{~Hz}, 3 \mathrm{H}), 1.20$ (d, J $5.1 \mathrm{~Hz}, 3 \mathrm{H})$.

${ }^{13}$ C NMR (75 MHz, $\left.\mathrm{CDCl}_{3}\right) \delta 122.9(\mathrm{C}), 79.3(\mathrm{CH}), 77.9(\mathrm{CH}), 45.1(\mathrm{C}), 41.2\left(\mathrm{CH}_{2}\right), 41.1\left(\mathrm{CH}_{2}\right)$, $35.4(\mathrm{CH}), 35.2(\mathrm{CH}), 17.0\left(\mathrm{CH}_{3}\right), 16.7\left(\mathrm{CH}_{3}\right)$.

IR $(\mathrm{KBr}) v_{\max } 2973,2933,2873,1426,1375,1351,1303,1238,1149,1122,1084,997,744 \mathrm{~cm}^{-1}$.

EI MS $m / z(70 \mathrm{eV}) 247$ and $245\left[(\mathrm{M}-\mathrm{Br} \bullet)^{+}\right.$, both 68\%], 175 and 173 (24 and 23), 147 and 145 (63 and 60), 114 (70), 65 (69), 55 (82), 43 (100).

HRMS Found: $(\mathrm{M}-\mathrm{Br} \bullet)^{+}, 245.0170 . \mathrm{C}_{10} \mathrm{H}_{14}{ }^{79} \mathrm{Br}_{2} \mathrm{O}_{2}$ requires $(\mathrm{M}-\mathrm{Br} \bullet)^{+}, 245.0177$.

Specific rotation $[\alpha]_{\mathrm{D}}-2.1\left(c 0.9, \mathrm{CHCl}_{3}\right)$.

\section{6,6-Dibromobicyclo[3.1.0]hexan-3-one (2R,3R)-2,3-Dibenzylethylene Ketal (22)}

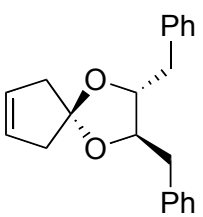

20

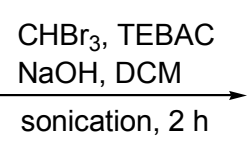

sonication, $2 \mathrm{~h}$

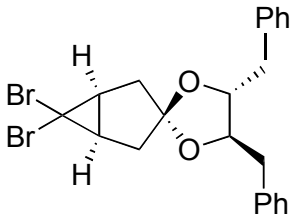

22

Alkene 20 (78 $\mathrm{mg}, 0.69 \mathrm{mmol}$ ) was subjected to dibromocarbene addition under the same conditions as employed for the conversion $19 \rightarrow 21$ described immediately above. The brown oil obtained on workup was subjected to flash chromatography (silica, 1:25 v/v ethyl acetate/hexane) and concentration of the relevant fractions $\left(R_{\mathrm{f}} 0.4\right.$ in $1: 5: 11 \mathrm{v} / \mathrm{v}$ ethyl acetate/DCM/hexane $)$ afforded the title cyclopropane 22 (95 $\mathrm{mg}, 78 \%)$ as a clear, colorless oil. 
${ }^{1}$ H NMR $\left(300 \mathrm{MHz}, \mathrm{CDCl}_{3}\right.$ ) $\delta$ 7.35-7.21 (complex m, 6 H), 7.19-7.10 (complex m, 4 H), 3.87 (m, $2 \mathrm{H}), 2.87-2.73$ (complex m, $2 \mathrm{H}$ ), 2.68-2.55 (complex m, $2 \mathrm{H}$ ), 2.31-2.12 (complex m, $4 \mathrm{H}$ ), 1.93-1.72 (complex m, $2 \mathrm{H}$ ).

${ }^{13}$ C NMR (75 MHz, $\left.\mathrm{CDCl}_{3}\right) \delta 137.2(\mathrm{C}), 137.1(\mathrm{C}), 129.7(\mathrm{CH}), 129.6(\mathrm{CH}), 128.6(\mathrm{CH}), 128.5$ $(\mathrm{CH}), 126.8(\mathrm{CH}), 126.8(\mathrm{CH}), 123.4(\mathrm{C}), 81.5(\mathrm{CH}), 80.2(\mathrm{CH}), 44.8(\mathrm{C}), 41.1\left(\mathrm{CH}_{2}\right), 41.0\left(\mathrm{CH}_{2}\right)$, $39.3\left(\mathrm{CH}_{2}\right), 38.9\left(\mathrm{CH}_{2}\right), 35.6(\mathrm{CH}), 35.2(\mathrm{CH})$.

IR $(\mathrm{KBr}) v_{\max } 3062,3028,2932,1603,1496,1454,1351,1305,1239,1146,1116,1078,1031$, $745,700 \mathrm{~cm}^{-1}$.

EI MS m/z (70 eV) 399 and $397\left[(\mathrm{M}-\mathrm{Br} \bullet)^{+}\right.$, both 90\%], 293 (45), 266 (92), 207 (63), 133 (100), 129 (85), 105 (76), 91 (87), 65 (84).

HRMS Found: $(\mathrm{M}-\mathrm{Br} \bullet)^{+}, 397.0807 . \mathrm{C}_{22} \mathrm{H}_{22}{ }^{79} \mathrm{Br}_{2} \mathrm{O}_{2}$ requires $(\mathrm{M}-\mathrm{Br} \bullet)^{+}, 397.0803$.

Specific rotation $[\alpha]_{\mathrm{D}}+3.2\left(c 0.5, \mathrm{CHCl}_{3}\right)$.

$(2 ' R, 3 ' R, 7 ' S)-3-\left\{8^{\prime}-\right.$ Bromo-2',3'-dimethyl-1',4'-dioxaspiro[4.5]dec-8'-en-7'-yl $\}$-indole

(23a)

and $\left(2 ' R, 3 ' R, 7^{\prime} R\right)-3-\left\{8^{\prime}-B r o m o-2 ', 3 '-d i m e t h y l-1 ', 4^{\prime}\right.$-dioxaspiro-[4.5]dec-8'-en-7'-yl\}indole (23b)

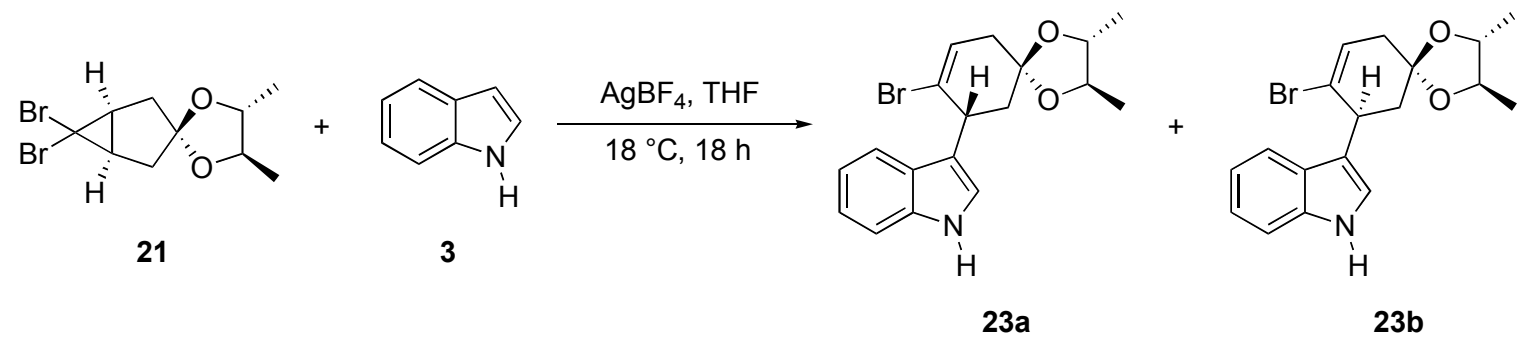

Silver tetrafluoroborate $(270 \mathrm{mg}, 1.39 \mathrm{mmol})$ was added, in one portion, to a magnetically stirred solution of ketal 21 (225 mg, $0.69 \mathrm{mmol})$ and indole (3) $(85 \mathrm{mg}, 0.72 \mathrm{mmol})$ in anhydrous THF $(25 \mathrm{~mL})$ maintained under a nitrogen atmosphere. The ensuing mixture was immediately protected from light and stirring continued at room temperature for $18 \mathrm{~h}$. The reaction mixture was then filtered through a plug of Celite ${ }^{\mathrm{TM}}$ that was washed with ethyl acetate $(50 \mathrm{~mL})$. The combined filtrates were concentrated under reduced pressure and the ensuing residue subjected to flash chromatography (silica, 1:10 v/v ethyl acetate/hexane elution). Concentration of the relevant fractions $\left(R_{\mathrm{f}} 0.3\right.$ in 2:5:11 v/v/v ethyl acetate/DCM/hexane) afforded a ca. 1:1 mixture of the title 
products, 23a and 23b (148 $\mathrm{mg}, 59 \%)$ as an oily solid. Subjection of a portion of this mixture to semi-preparative HPLC (YMC ODS-AQ $5 \mu \mathrm{m} 250 \times 20 \mathrm{~mm}$ column using 3:2 v/v MeOH/ $\mathrm{H}_{2} \mathrm{O}$ as the eluting solvent and a flow rate of $15 \mathrm{ml} / \mathrm{min}$ ) afforded two fractions, A and $\mathrm{B}$.

Concentration of fraction A ( $\left.R_{\mathrm{f}} 46.7 \mathrm{~min}\right)$ afforded a white crystalline solid, $\mathrm{mp} 58-61{ }^{\circ} \mathrm{C}$, tentatively identified as indole $\mathbf{2 3 a}$.

${ }^{1}$ H NMR $\left(300 \mathrm{MHz}, \mathrm{CDCl}_{3}\right) \delta 8.06(\operatorname{broad~s}, 1 \mathrm{H}), 7.67(\mathrm{~d}, J 8.1 \mathrm{~Hz}, 1 \mathrm{H}), 7.40(\mathrm{~d}, J 8.1 \mathrm{~Hz}, 1 \mathrm{H})$, $7.24(\mathrm{t}, J 8.1 \mathrm{~Hz}, 1 \mathrm{H}), 7.18(\mathrm{~d}, J 8.1 \mathrm{~Hz}, 1 \mathrm{H}), 7.12(\mathrm{~d}, J 2.2 \mathrm{~Hz}, 1 \mathrm{H}), 6.23(\mathrm{dt}, J 5.2$ and $2.4 \mathrm{~Hz}, 1$ H), $4.23(\mathrm{~m}, 1 \mathrm{H}), 3.85-3.70$ (complex m, $2 \mathrm{H}), 2.63$ (dd, $J 17.3$ and $3.3 \mathrm{~Hz}, 1 \mathrm{H}), 2.51-2.39$ (complex m, $2 \mathrm{H}), 2.25(\mathrm{~m}, 1 \mathrm{H}), 1.36(\mathrm{~d}, J 5.6 \mathrm{~Hz}, 3 \mathrm{H}), 1.16(\mathrm{~d}, J 5.6 \mathrm{~Hz}, 3 \mathrm{H})$.

${ }^{13}$ C NMR (75 MHz, $\left.\mathrm{CDCl}_{3}\right) \delta 136.5(\mathrm{C}), 127.2(\mathrm{CH}), 126.5(\mathrm{C}), 122.7(\mathrm{CH}), 122.1(\mathrm{CH}), 119.5$ $(\mathrm{CH}), 119.3(\mathrm{CH}), 117.5(\mathrm{C}), 111.5(\mathrm{CH}), 106.2(\mathrm{C}), 78.8(\mathrm{CH}), 78.7(\mathrm{CH}), 41.7\left(\mathrm{CH}_{2}\right), 41.2(\mathrm{CH})$, $39.7\left(\mathrm{CH}_{2}\right), 17.4\left(\mathrm{CH}_{3}\right), 17.1\left(\mathrm{CH}_{3}\right)$ (one signal, due to a quaternary carbon, obscured or overlapping).

IR (KBr) $v_{\max } 3419,2971,2929,2874,1619,1456,1357,1294,1244,1141,1111,1082,1024$, $742 \mathrm{~cm}^{-1}$.

EI MS $m / z(70 \mathrm{eV}) 363$ and $361\left(\mathrm{M}^{+\bullet}\right.$, both $\left.76 \%\right), 249$ and 247 (84 and 85), $168(100), 167(81)$, 115 (83), 84 (44), 43 (42).

HRMS Found: $\mathrm{M}^{+\bullet}$, 361.0680. $\mathrm{C}_{18} \mathrm{H}_{20}{ }^{79} \mathrm{BrNO}_{2}$ requires $\mathrm{M}^{+\bullet}, 361.0677$;

Found: $\mathrm{M}^{+\bullet}, 363.0669 . \mathrm{C}_{18} \mathrm{H}_{20}{ }^{81} \mathrm{BrNO}_{2}$ requires $\mathrm{M}^{+\bullet}, 363.0657$.

Specific rotation $[\alpha]_{\mathrm{D}}-92.5\left(\right.$ c $\left.0.4, \mathrm{CHCl}_{3}\right)$.

Concentration of fraction $\mathrm{B}\left(R_{\mathrm{f}} 48.3 \mathrm{~min}\right)$ afforded a white solid, mp $99-101{ }^{\circ} \mathrm{C}$, tentatively identified as indole $\mathbf{2 3 b}$.

${ }^{1} \mathbf{H}$ NMR $\left(300 \mathrm{MHz}, \mathrm{CDCl}_{3}\right) \delta 8.02(\operatorname{broad~s}, 1 \mathrm{H}), 7.63(\mathrm{~d}, J 8.1 \mathrm{~Hz}, 1 \mathrm{H}), 7.37(\mathrm{~d}, J 8.1 \mathrm{~Hz}, 1 \mathrm{H})$, $7.21(\mathrm{t}, J 8.1 \mathrm{~Hz}, 1 \mathrm{H}), 7.15$ (t, $J 8.1 \mathrm{~Hz}, 1 \mathrm{H}), 7.10(\mathrm{~m}, 1 \mathrm{H}), 6.17$ (dt, $J 5.2$ and $2.4 \mathrm{~Hz}, 1 \mathrm{H}), 4.21$ (m, $1 \mathrm{H}$ ), 3.75-3.57 (complex m, $2 \mathrm{H}$ ), 2.63 (dd, $J 16.6$ and $6.6 \mathrm{~Hz}, 1 \mathrm{H}$ ), 2.42-2.29 (complex m, 2 H), $2.17(\mathrm{~m}, 1 \mathrm{H}), 1.31(\mathrm{~d}, J 5.9 \mathrm{~Hz}, 3 \mathrm{H}), 1.24(\mathrm{~d}, J 5.9 \mathrm{~Hz}, 3 \mathrm{H})$.

${ }^{13} \mathrm{C}$ NMR $\left(75 \mathrm{MHz}, \mathrm{CDCl}_{3}\right) \delta 136.5(\mathrm{C}), 127.5(\mathrm{CH}), 127.0(\mathrm{C}), 126.5(\mathrm{C}), 122.6(\mathrm{CH}), 122.2$ $(\mathrm{CH}), 119.6(\mathrm{CH}), 119.4(\mathrm{CH}), 117.7(\mathrm{C}), 111.5(\mathrm{CH}), 106.1(\mathrm{C}), 78.6(\mathrm{CH}), 78.2(\mathrm{CH}), 42.5$ $\left(\mathrm{CH}_{2}\right), 41.1(\mathrm{CH}), 39.1\left(\mathrm{CH}_{2}\right), 17.0\left(\mathrm{CH}_{3}\right), 16.8\left(\mathrm{CH}_{3}\right)$. 
IR $(\mathrm{KBr}) \boldsymbol{v}_{\max } 3421,2970,2927,1619,1456,1375,1356,1292,1244,1142,1111,1083,1022$, $741 \mathrm{~cm}^{-1}$.

EI MS $m / z(70 \mathrm{eV}) 363$ and $361\left(\mathrm{M}^{+\bullet}\right.$, both 42\%), 249 and 247 (60 and 58), 168 (100), 167 (61), $115(55), 43(27)$.

HRMS Found: $\mathrm{M}^{+\bullet}, 361.0674 . \mathrm{C}_{18} \mathrm{H}_{20}{ }^{79} \mathrm{BrNO}_{2}$ requires $\mathrm{M}^{+\bullet}, 361.0677$;

Found: $\mathrm{M}^{+\bullet}, 363.0659 . \mathrm{C}_{18} \mathrm{H}_{20}{ }^{81} \mathrm{BrNO}_{2}$ requires $\mathrm{M}^{+\bullet}, 363.0657$.

Specific rotation $[\alpha]_{\mathrm{D}}+110.5\left(c 0.4, \mathrm{CHCl}_{3}\right)$.

$(2 ' R, 3 ' R, 7 ' S)-3-\left\{8^{\prime}\right.$-Bromo-2,3-dibenzyl-1',4'-dioxaspiro[4.5]dec-8'-en-7'yl\}-indole (24a) and $\left(2 ' R, 3 ' R, 7^{\prime} R\right)-3-\left\{8^{\prime}\right.$-Bromo-2,3-dibenzyl-1',4'-dioxaspiro[4.5]dec-8'-en-7'yl\}indole (24b)

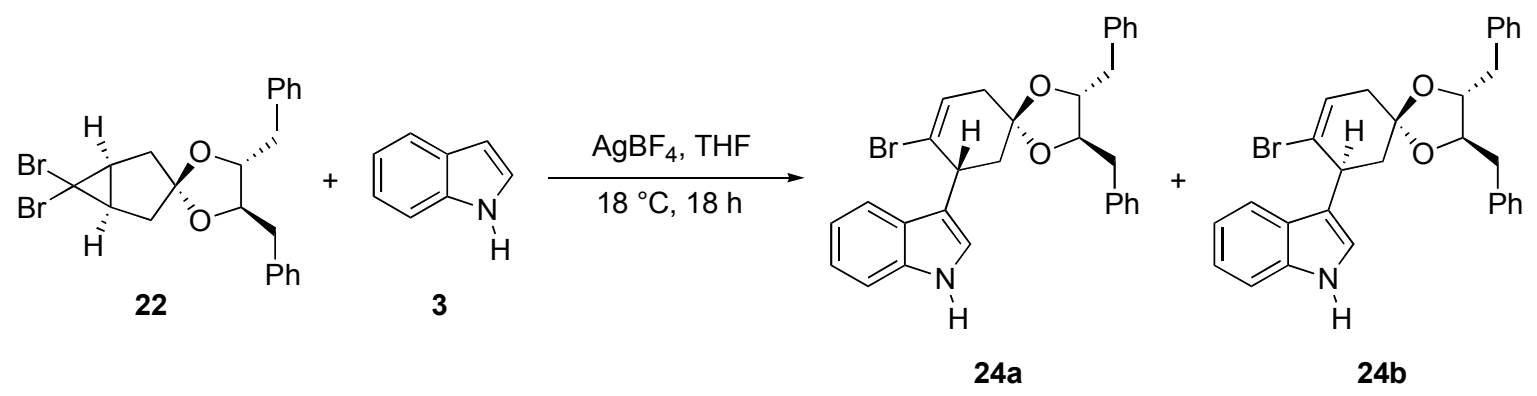

Cyclopropane 22 (95 mg, $0.20 \mathrm{mmol}$ ) was subjected to reaction with silver tetrafluoroborate (77 $\mathrm{mg}, 0.40 \mathrm{mmol})$ in the presence of indole (3) $(24 \mathrm{mg}, 0.20 \mathrm{mmol})$ under the same conditions as employed for the conversion $21 \rightarrow$ 23a + 23b described immediately above. The brown oil obtained on workup was subjected to flash chromatography (silica, 1:10 v/v ethyl acetate/hexane elution) and concentration of the relevant fractions $\left(R_{\mathrm{f}} \quad 0.4\right.$ in $2: 5: 11 \mathrm{v} / \mathrm{v} / \mathrm{v}$ ethyl acetate/DCM/hexane) afforded a ca. 1:1 mixture of the title products, $\mathbf{2 4 a}$ and $\mathbf{2 4 b}(71 \mathrm{mg}, 70 \%)$ as an oily solid. Subjection of a portion of this mixture to semi-preparative HPLC ( $\mu$ Porasil ${ }^{\circledR}$ Silica $10 \mu \mathrm{m} 300 \times 19 \mathrm{~mm}$ column using $0.2 \%$ isopropanol in hexane as the eluting solvent and a flow rate of $15 \mathrm{ml} / \mathrm{min}$ ) afforded two fractions, $\mathrm{A}$ and $\mathrm{B}$.

Concentration of A ( $\left.R_{\mathrm{f}} 27.5 \mathrm{~min}\right)$ afforded a white solid, $\mathrm{mp} 110-112{ }^{\circ} \mathrm{C}$, tentatively identified as indole 24a.

${ }^{1}$ H NMR $\left(300 \mathrm{MHz}, \mathrm{CDCl}_{3}\right) \delta 7.99$ (broad s, $\left.1 \mathrm{H}\right), 7.60(\mathrm{~d}, J 8.0 \mathrm{~Hz}, 1 \mathrm{H}), 7.37(\mathrm{~d}, J 8.0 \mathrm{~Hz}, 1 \mathrm{H})$, 
7.35-7.08 (complex m, $10 \mathrm{H}), 7.07$ (d, J 2.5 Hz, $1 \mathrm{H}), 6.95$ (m, $2 \mathrm{H}), 6.16(\mathrm{~m}, 1 \mathrm{H}), 4.15$ (m, $1 \mathrm{H})$, $3.97(\mathrm{~m}, 2 \mathrm{H}), 2.91(\mathrm{dd}, J 14.0$ and $5.2 \mathrm{~Hz}, 1 \mathrm{H}), 2.72(\mathrm{dd}, J 14.0$ and $5.0 \mathrm{~Hz}, 1 \mathrm{H}), 2.63-2.52$ (complex m, 2 H), 2.49-2.25 (complex m, $3 \mathrm{H}$ ), 2.18 (ddd, $J$ 13.3, 6.3 and $2.3 \mathrm{~Hz}, 1 \mathrm{H}$ ).

${ }^{13}$ C NMR (75 MHz, $\left.\mathrm{CDCl}_{3}\right) \delta 137.4(\mathrm{C}), 137.3(\mathrm{C}), 136.5(\mathrm{C}), 129.8(\mathrm{CH}), 129.5(\mathrm{CH}), 128.5$ $(\mathrm{CH}), 128.4(\mathrm{CH}), 127.4(\mathrm{CH}), 126.8(\mathrm{CH}), 126.6(\mathrm{CH}), 126.5(\mathrm{C}), 126.0(\mathrm{C}), 122.8(\mathrm{CH}), 122.2$ $(\mathrm{CH}), 119.6(\mathrm{CH}), 119.3(\mathrm{CH}), 117.4(\mathrm{C}), 111.5(\mathrm{CH}), 106.7(\mathrm{C}), 81.0(\mathrm{CH}), 80.9(\mathrm{CH}), 41.7$ $\left(\mathrm{CH}_{2}\right), 41.3(\mathrm{CH}), 39.5\left(\mathrm{CH}_{2}\right), 39.3\left(\mathrm{CH}_{2}\right)$ (one signal due to $\mathrm{CH}_{2}$ obscured or overlapping).

IR $(\mathrm{KBr}) v_{\max } 3427,3027,2928,1495,1455,1358,1244,1115,1046,909,741,701 \mathrm{~cm}^{-1}$.

EI MS $m / z(70 \mathrm{eV}) 515$ and $513\left(\mathrm{M}^{+\bullet}\right.$, both 15\%), 267 (27), 249 and 247 (20 and 21), 168 (33), 167 (21), 105 (15), 91 (42), 78 (83), 63 (100), 45 (30).

HRMS Found: $\mathrm{M}^{+\bullet}, 513.1323 . \mathrm{C}_{30} \mathrm{H}_{28}{ }^{79} \mathrm{BrNO}_{2}$ requires $\mathrm{M}^{+\bullet}, 513.1303$;

Found: $\mathrm{M}^{+\bullet}, 515.1290 . \mathrm{C}_{30} \mathrm{H}_{28}{ }^{81} \mathrm{BrNO}_{2}$ requires $\mathrm{M}^{+\bullet}, 515.1283$.

Specific rotation $[\alpha]_{\mathrm{D}}-18.2\left(c 0.5, \mathrm{CHCl}_{3}\right)$.

Concentration of fraction $\mathrm{B}\left(R_{\mathrm{f}} 16.8 \mathrm{~min}\right)$ afforded a white solid, mp $111-114{ }^{\circ} \mathrm{C}$, tentatively identified as indole $\mathbf{2 4 b}$.

${ }^{1}$ H NMR $\left(300 \mathrm{MHz}, \mathrm{CDCl}_{3}\right) \delta 7.86$ (broad s, $\left.1 \mathrm{H}\right), 7.57$ (d, J 8.0 Hz, $1 \mathrm{H}$ ), 7.37-7.20 (complex m, 7 H), 7.19-7.12 (complex m, 3 H), 7.11-7.06 (complex m, 3 H), 6.99 (d, J 2.3 Hz, 1 H), 6.14 (m, 1 H), $4.16(\mathrm{~m}, 1 \mathrm{H}), 3.98(\mathrm{~m}, 1 \mathrm{H}), 3.85(\mathrm{~m}, 1 \mathrm{H}), 2.91(\mathrm{dd}, J 13.9$ and $5.6 \mathrm{~Hz}, 1 \mathrm{H}), 2.73(\mathrm{~m}, 2 \mathrm{H})$, $2.61(\mathrm{~m}, 1 \mathrm{H}), 2.56$ (m, $1 \mathrm{H}), 2.35-2.23$ (complex m, $2 \mathrm{H}), 2.01$ (ddd, J 13.2, 6.2 and $2.3 \mathrm{~Hz}, 1 \mathrm{H}$ ).

${ }^{13}$ C NMR (75 MHz, $\left.\mathrm{CDCl}_{3}\right) \delta 137.6(\mathrm{C}), 137.1(\mathrm{C}), 136.4(\mathrm{C}), 129.8(\mathrm{CH}), 129.6(\mathrm{CH}), 128.6$ $(\mathrm{CH}), 128.5(\mathrm{CH}), 127.4(\mathrm{CH}), 126.8(\mathrm{CH}), 126.6(\mathrm{CH}), 126.5(\mathrm{C}), 126.4(\mathrm{C}), 122.8(\mathrm{CH}), 122.1$ $(\mathrm{CH}), 119.5(\mathrm{CH}), 119.2(\mathrm{CH}), 117.3(\mathrm{C}), 111.4(\mathrm{CH}), 106.6(\mathrm{C}), 80.8(\mathrm{CH}), 80.4(\mathrm{CH}), 41.9$ $\left(\mathrm{CH}_{2}\right), 41.0(\mathrm{CH}), 39.2(4)\left(\mathrm{CH}_{2}\right), 39.2(1)\left(\mathrm{CH}_{2}\right), 39.0\left(\mathrm{CH}_{2}\right)$.

IR (KBr) $v_{\max } 3428,3027,2918,1495,1455,1357,1290,1244,1109,1046,909,741,701 \mathrm{~cm}^{-1}$.

EI MS $m / z(70 \mathrm{eV}) 515$ and $513\left(\mathrm{M}^{+\bullet}\right.$, both 35\%), 267 (57), 249 and 247 (44 and 45), 168 (70), 167 (48), 105 (43), 91 (82), 69 (100), 57 (96), 43 (86).

HRMS Found: $\mathrm{M}^{+\bullet}$, 513.1303. $\mathrm{C}_{30} \mathrm{H}_{28}{ }^{79} \mathrm{BrNO}_{2}$ requires $\mathrm{M}^{+\bullet}, 513.1303$;

Found: $\mathrm{M}^{+\bullet}, 515.1304 . \mathrm{C}_{30} \mathrm{H}_{28}{ }^{81} \mathrm{BrNO}_{2}$ requires $\mathrm{M}^{+\bullet}, 515.1283$.

Specific rotation $[\alpha]_{\mathrm{D}}+24.0\left(c 0.5, \mathrm{CHCl}_{3}\right)$. 


\section{3-(2-Bromophenyl)indole (25)}

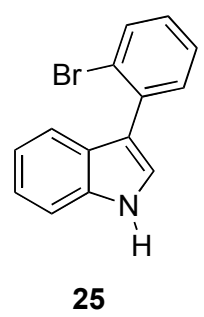

Samples of compounds 23a, 23b, 24a and/or 24b left standing for prolonged periods at room temperature eventually rearranged to give a dark brown oil. Subjection of such material to flash chromatography (silica, 1:19.5:79.5 v/v/v triethylamine/ethyl acetate/hexane elution) afforded, after concentration of the relevant fractions $\left(R_{\mathrm{f}} 0.5\right)$, compound 25 in varying yields and as a paleorange oil.

${ }^{1}$ H NMR (300 MHz) $\delta 8.29$ (broad s, $\left.1 \mathrm{H}\right), 7.89$ (dd, J 7.8 and $\left.0.6 \mathrm{~Hz}, 1 \mathrm{H}\right), 7.56$ (broad s, $\left.1 \mathrm{H}\right)$, 7.45 (dm, J 7.8 Hz, $1 \mathrm{H}$ ), 7.37-7.16 (complex m, $4 \mathrm{H}$ ), 6.76-6.68 (complex m, 2 H).

${ }^{13}$ C NMR (75 MHz) $\delta 134.4(\mathrm{C}), 132.3(\mathrm{CH}), 131.7(\mathrm{CH}), 131.4(\mathrm{C}), 128.8(\mathrm{CH}), 122.5(\mathrm{CH})$, $121.8(\mathrm{CH}), 120.5(\mathrm{CH}), 120.2(\mathrm{C}), 119.5(1)(\mathrm{C}), 119.4(5)(\mathrm{CH}), 117.1(\mathrm{CH}), 112.7(\mathrm{C}), 114.4$ $(\mathrm{CH})$.

IR $v_{\max }$ (neat) $3410,2924,2852,1456,1260,1095,1013,800,743 \mathrm{~cm}^{-1}$.

Mass Spectrum (EI, 70eV) m/z 273 and $271\left(\mathrm{M}^{+*}\right.$, both 55\%), 149 (53), 97 (56), 95 (52), 83 (65), 81 (80), 69 (100), 57 (95), 55 (85), 43 (100), 41 (80).

HRMS Found $\mathrm{M}^{+\bullet}, 270.9998 . \mathrm{C}_{14} \mathrm{H}_{10}{ }^{79} \mathrm{BrN}$ requires $\mathrm{M}^{+\bullet}, 270.9997$. 


\section{X-ray Crystallographic Data for Compounds 8, 13a and 13b}

Crystal data for 8: $\mathrm{C}_{17} \mathrm{H}_{19} \mathrm{NO}_{2}, M=269.34, T=200 \mathrm{~K}$, orthorhombic, space group Pna ${ }_{1}, Z=8, a$ $=20.5365(5), b=5.2508(1), c=26.4263(6) \AA, V=2849.63(11) \AA^{3}, D_{x}=1.256 \mathrm{~g} \mathrm{~cm}^{-3}, 2872$ unique data $\left(\theta_{\max }=26.0^{\circ}\right), 2244$ with $I>1.5 \sigma(I) ; R=0.0381, R w=0.0468, S=1.1106$.

Crystal data for 13a: $\mathrm{C}_{18} \mathrm{H}_{23} \mathrm{~N}, M=253.39, T=200 \mathrm{~K}$, triclinic, space group $P \overline{\mathrm{I}}, Z=4, a=$ 7.7435(3), $b=10.6570(3), c=18.3316(4) \AA, \alpha=95.4430(18)^{\circ}, \beta=97.0565(18)^{\circ}, \gamma=$ 103.8689(14) $)^{\circ}, V=1445.36(8) \AA^{3}, D_{x}=1.164 \mathrm{~g} \mathrm{~cm}^{-3}, 5101$ unique data $\left(\theta_{\max }=25.0^{\circ}\right), 3516$ with $I$ $>3.0 \sigma(I) ; R=0.0439, R w=0.0548, S=1.0935$.

Crystal data for 13b: $\mathrm{C}_{18} \mathrm{H}_{23} \mathrm{~N}, M=253.39, T=200 \mathrm{~K}$, triclinic, space group $P \overline{\mathrm{I}}, Z=4, a=$ 7.9681(2), $b=10.7207(3), c=18.0210(3) \AA, \alpha=96.5216(15)^{\circ}, \beta=98.1632(17)^{\circ}, \gamma=$ 104.2454(13) $)^{\circ}, V=1459.16(6) \AA^{3}, D_{x}=1.153 \mathrm{~g} \mathrm{~cm}^{-3}, 6687$ unique data $\left(\theta_{\max }=27.5^{\circ}\right), 4326$ with $I$ $>3.0 \sigma(I) ; R=0.0419, R w=0.0537, S=1.0874$.

\section{Structure Determination}

Images were measured on a Nonius Kappa CCD diffractometer $(\mathrm{MoK} \alpha$, graphite monochromator, $\lambda=0.71073 \AA$ ). Programs used for data collection, refinement and solving structures: COLLECT, ${ }^{7}$ Denzo/Scalepack, ${ }^{8}$ SIR92 ${ }^{9}$ and CRYSTALS.${ }^{10}$ Crystallographic Information Files (cifs) have been deposited at the Cambridge Crystallographic Data Centre (CCDC reference numbers: 8 - 610988, 13a - 610989 and 13b - 610990). These data can be obtained free-of-charge via www.ccdc.cam.ac.uk/data_request/cif, by emailing data_request@ccdc.cam.ac.uk, or by contacting The Cambridge Crystallographic Data Centre, 12 Union Road, Cambridge CB2 1EZ, UK; fax: +44 1223336033 . 


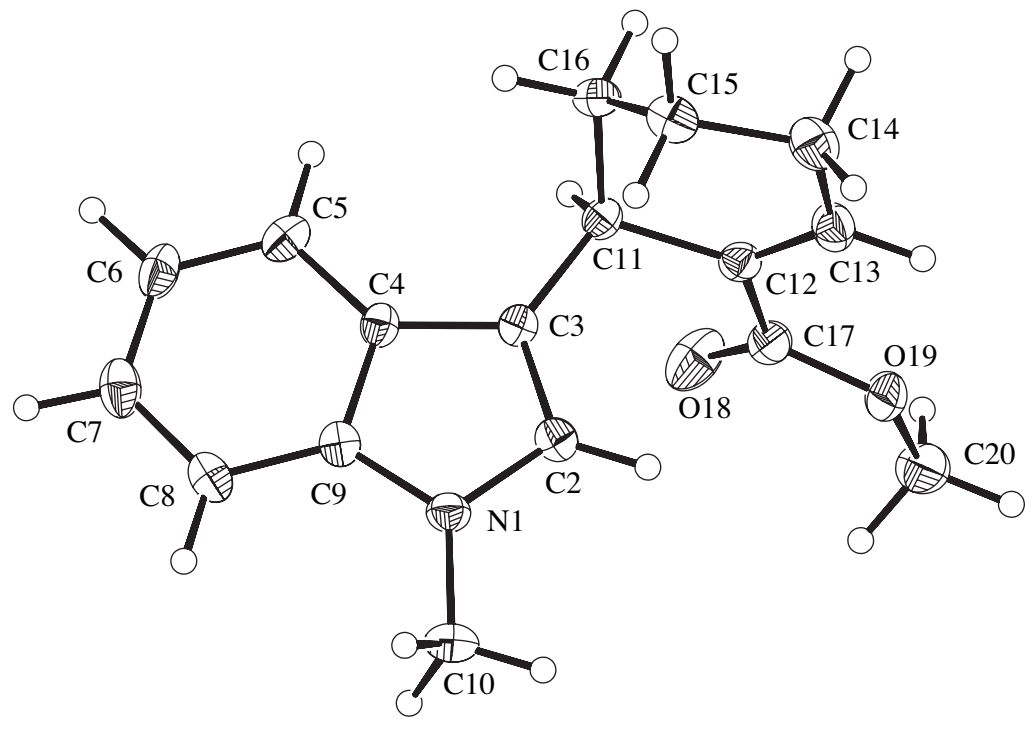

Figure S1. Structure of molecule one of $\mathrm{C}_{17} \mathrm{H}_{19} \mathrm{NO}_{2}(\mathbf{8})$ with labelling of non-hydrogen atoms. Anisotropic displacement ellipsoids show 30\% probability levels. Hydrogen atoms are drawn as circles with small radii.

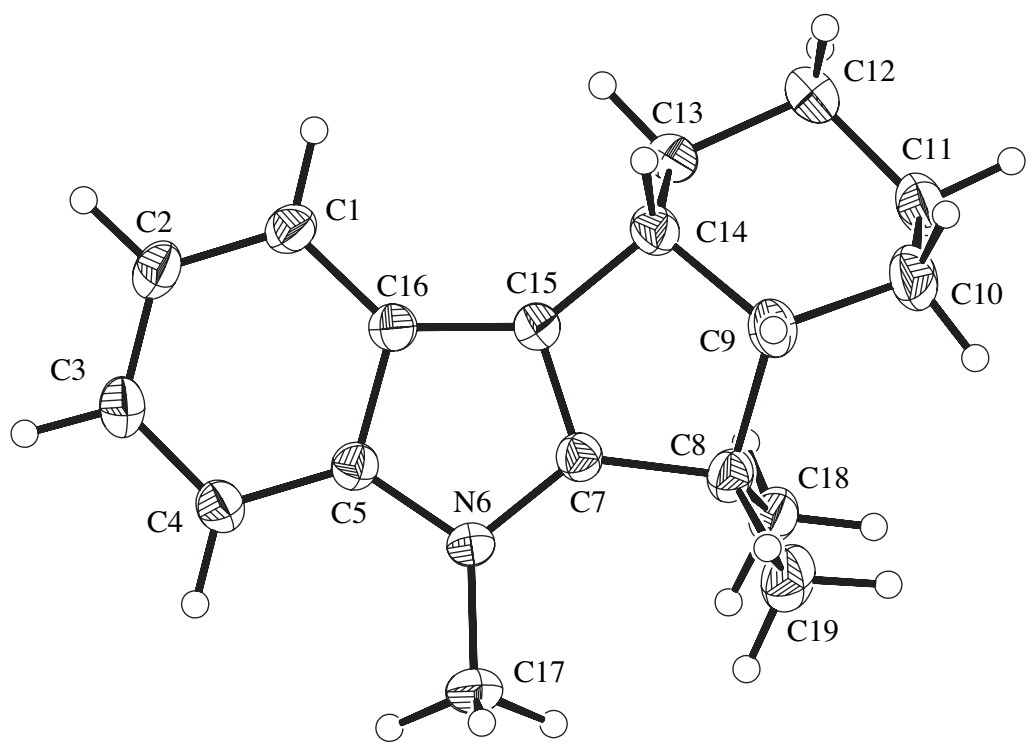

Figure S2. Structure of molecule one of $\mathrm{C}_{18} \mathrm{H}_{23} \mathrm{~N}$ (13a) with labelling of non-hydrogen atoms. Anisotropic displacement ellipsoids show $30 \%$ probability levels. Hydrogen atoms are drawn as circles with small radii. 


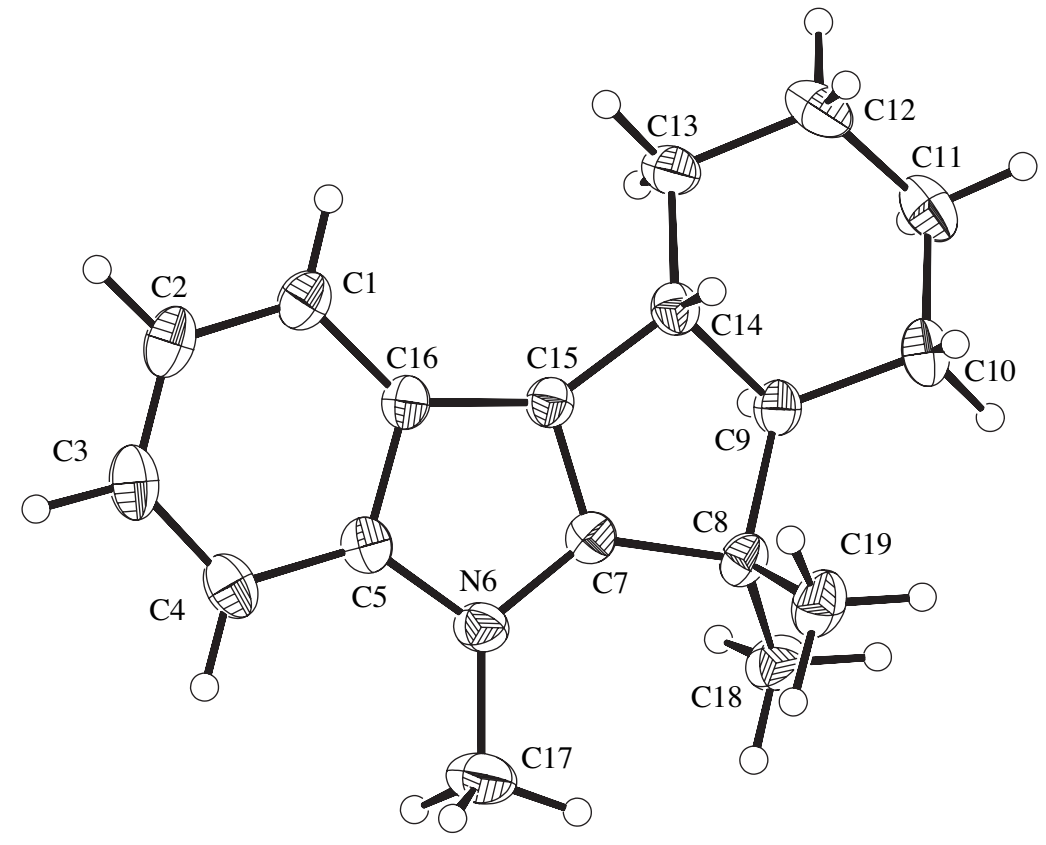

Figure S3. Structure of molecule one of $\mathrm{C}_{18} \mathrm{H}_{23} \mathrm{~N}(\mathbf{1 3 b})$ with labelling of non-hydrogen atoms. Only the major site of each disordered atom is shown. Anisotropic displacement ellipsoids show $30 \%$ probability levels. Hydrogen atoms are drawn as circles with small radii. 


\section{References}

1. Still, W. C.; Kahn, M.; Mitra, A. J. Org. Chem. 1978, 43, 2923.

2. Sonnenberg, J.; Winstein, S. J. Org. Chem. 1962, 27, 748.

3. Oster, T. A.; Harris, T. M. Tetrahedron Lett. 1983, 24, 1851.

4. Dreyfuss, M. P. J. Org. Chem. 1963, 28, 3269.

5. Rieke, R. D.; Daruwala, K. P.; Forkner, M. W. Organometallics 1991, 10, 2946.

6. Crotti, P.; Ferretti, M.; Macchia, F.; Stoppioni, A. J. Org. Chem. 1984, 49, 4706.

7. COLLECT. Nonius BV, Delft: The Netherlands, 1997.

8. DENZO-SMN. Otwinowski, Z.; Minor, W. Processing of X-ray diffraction data collected in oscillation mode. In Methods in Enzymology, Volume 276: Macromolecular Crystallography, Part A; Carter Jr., C. W.; Sweets, R. M., Eds.; Academic Press: New York, 1997; pp. 307326.

9. SIR92. Altomare, A.; Cascarano, G.; Giacovazzo, C.; Guagliardi, A.; Burla, M. C.; Polidori, G.; Camalli, M. J. Appl. Crystallogr. 1994, 27, 435.

10. Betteridge, P. W.; Carruthers, J. R.; Cooper, R. I.; Prout, K.; Watkin, D. J. J. Appl. Crystallogr. 2003, 36, 1487. 
$75 \mathrm{MHz}$ APT ${ }^{13} \mathrm{C}$ NMR Spectrum of Compound 6 (recorded in $\mathrm{CDCl}_{3}$ )

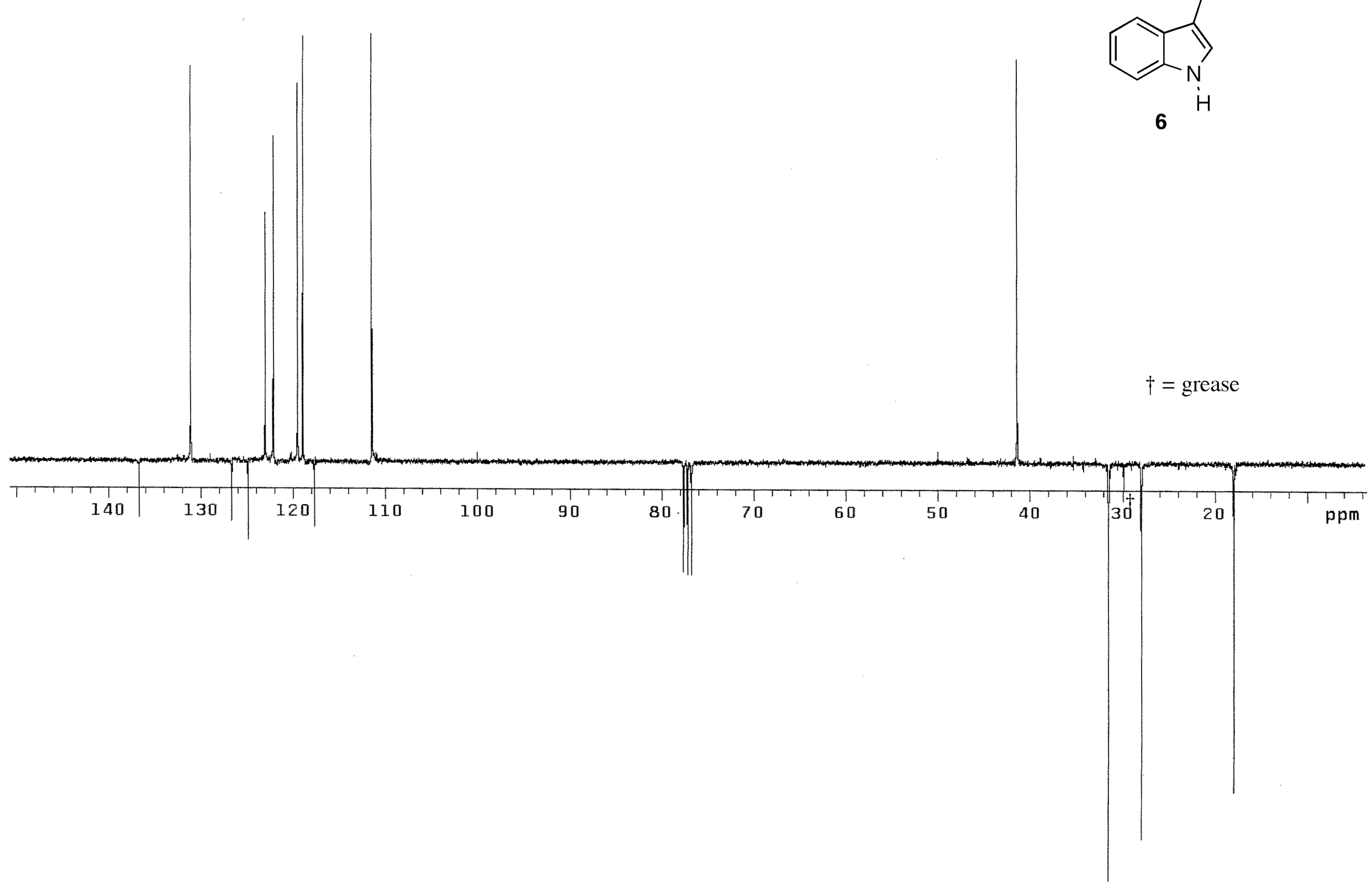


$75 \mathrm{MHz}$ APT ${ }^{13} \mathrm{C}$ NMR Spectrum of Compound 7 (recorded in $\mathrm{CDCl}_{3}$ )

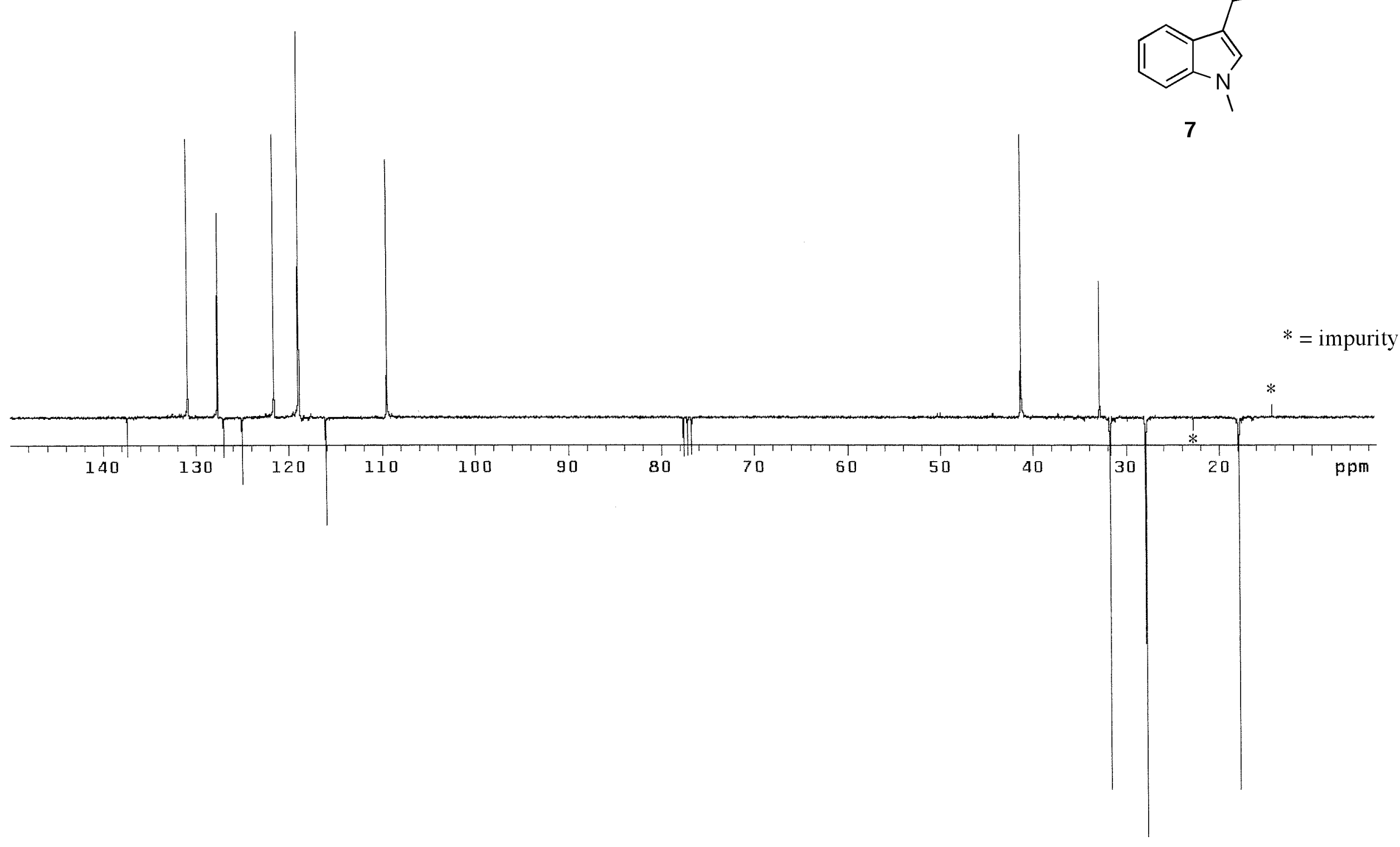


$300 \mathrm{MHz}{ }^{1} \mathrm{H}$ NMR Spectrum of Compound 8

(recorded in $\mathrm{CDCl}_{3}$ )

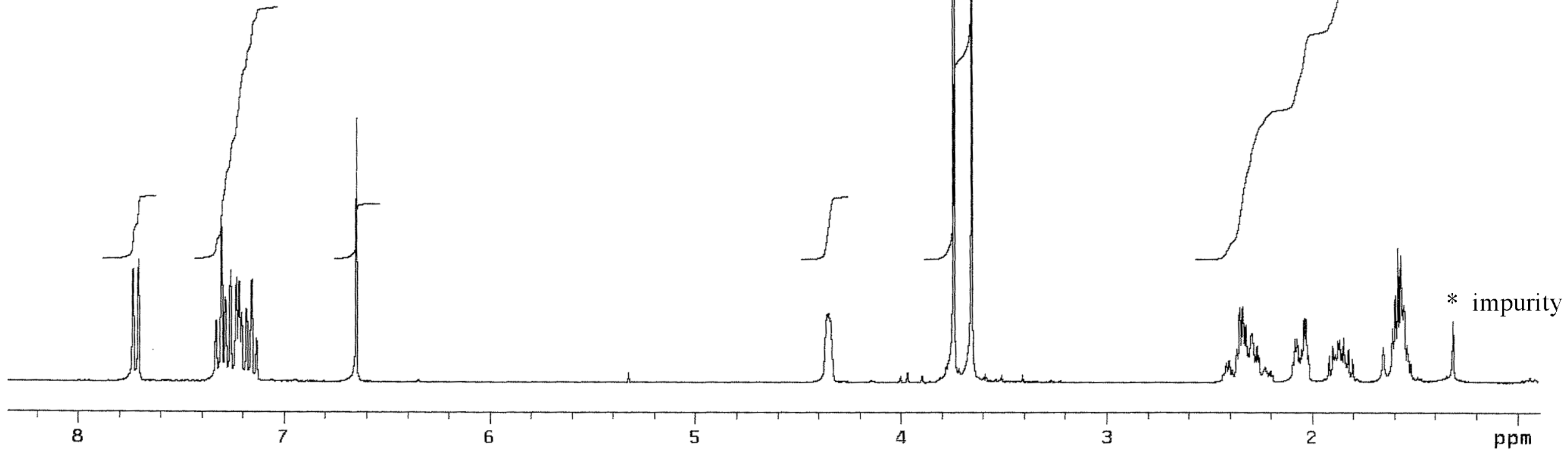


$300 \mathrm{MHz}{ }^{1} \mathrm{H}$ NMR Spectrum of Compound 9 (recorded in $\mathrm{CDCl}_{3}$ )

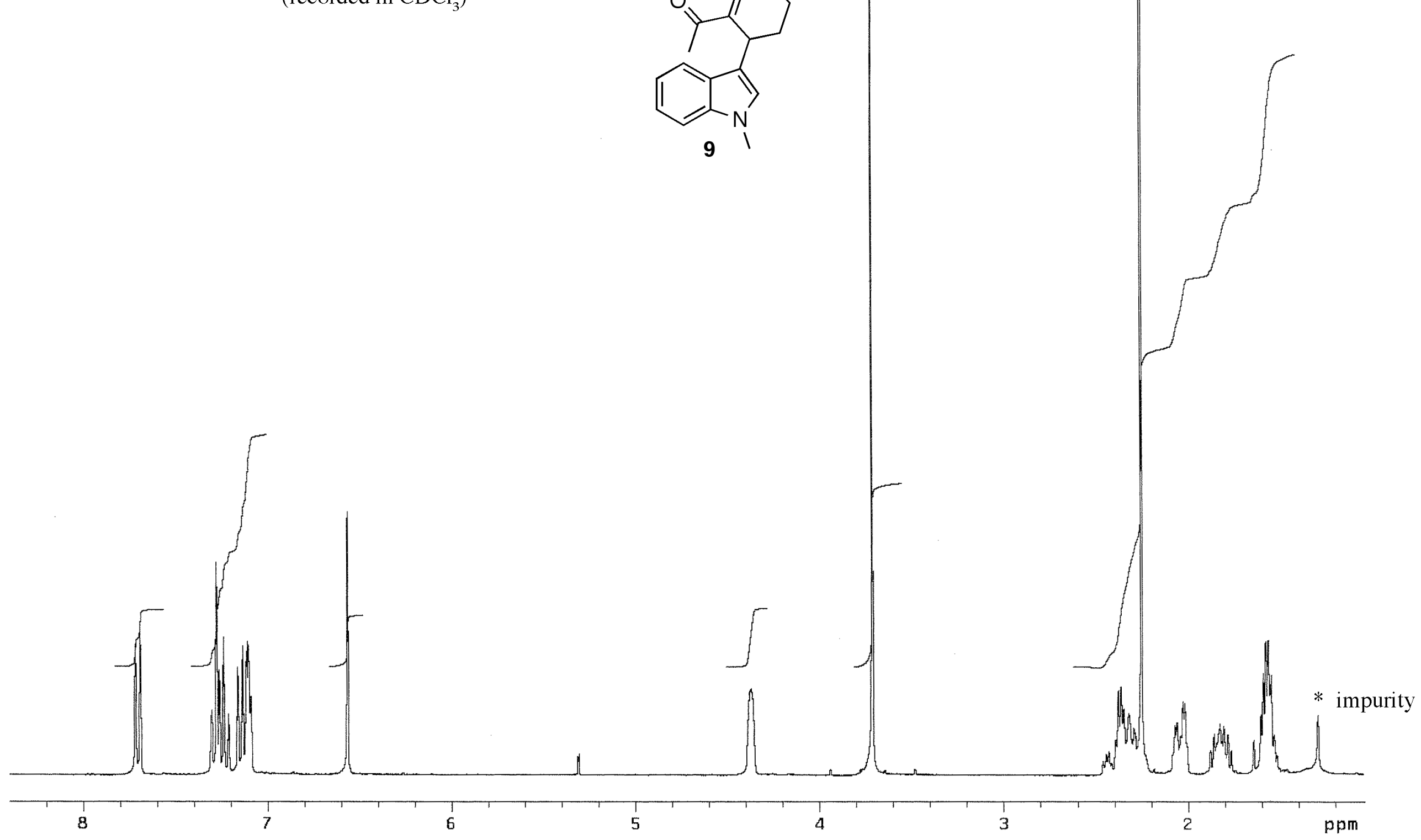


$75 \mathrm{MHz}$ APT ${ }^{13} \mathrm{C}$ NMR Spectrum of Compound 10a (recorded in $\mathrm{CDCl}_{3}$ )

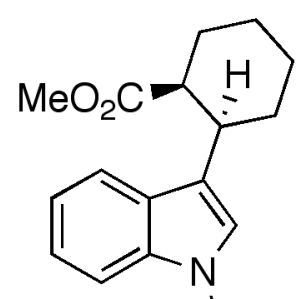

$10 \mathrm{a}$

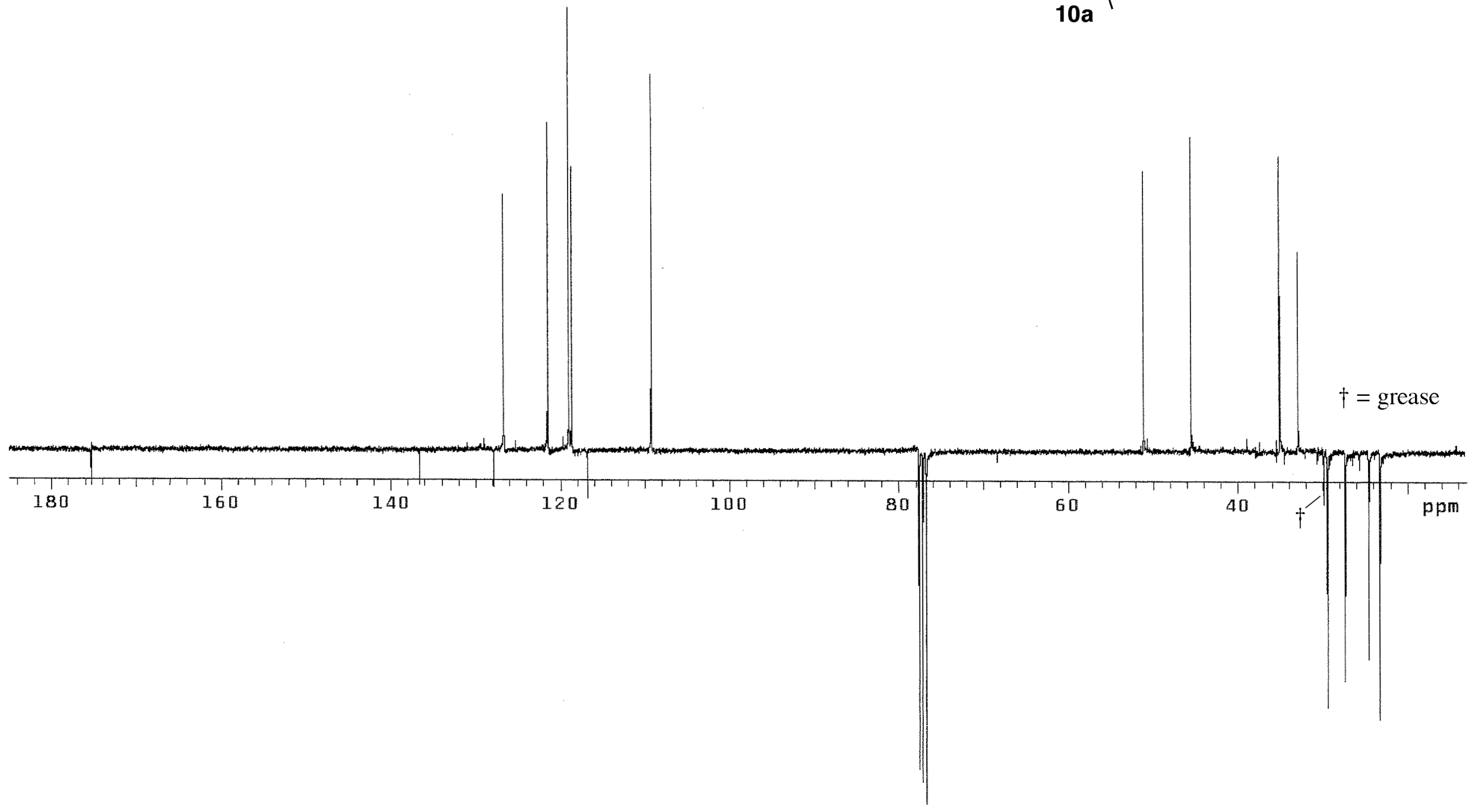


$75 \mathrm{MHz}$ APT ${ }^{13} \mathrm{C}$ NMR Spectrum of Compound 10b (recorded in $\mathrm{CDCl}_{3}$ )

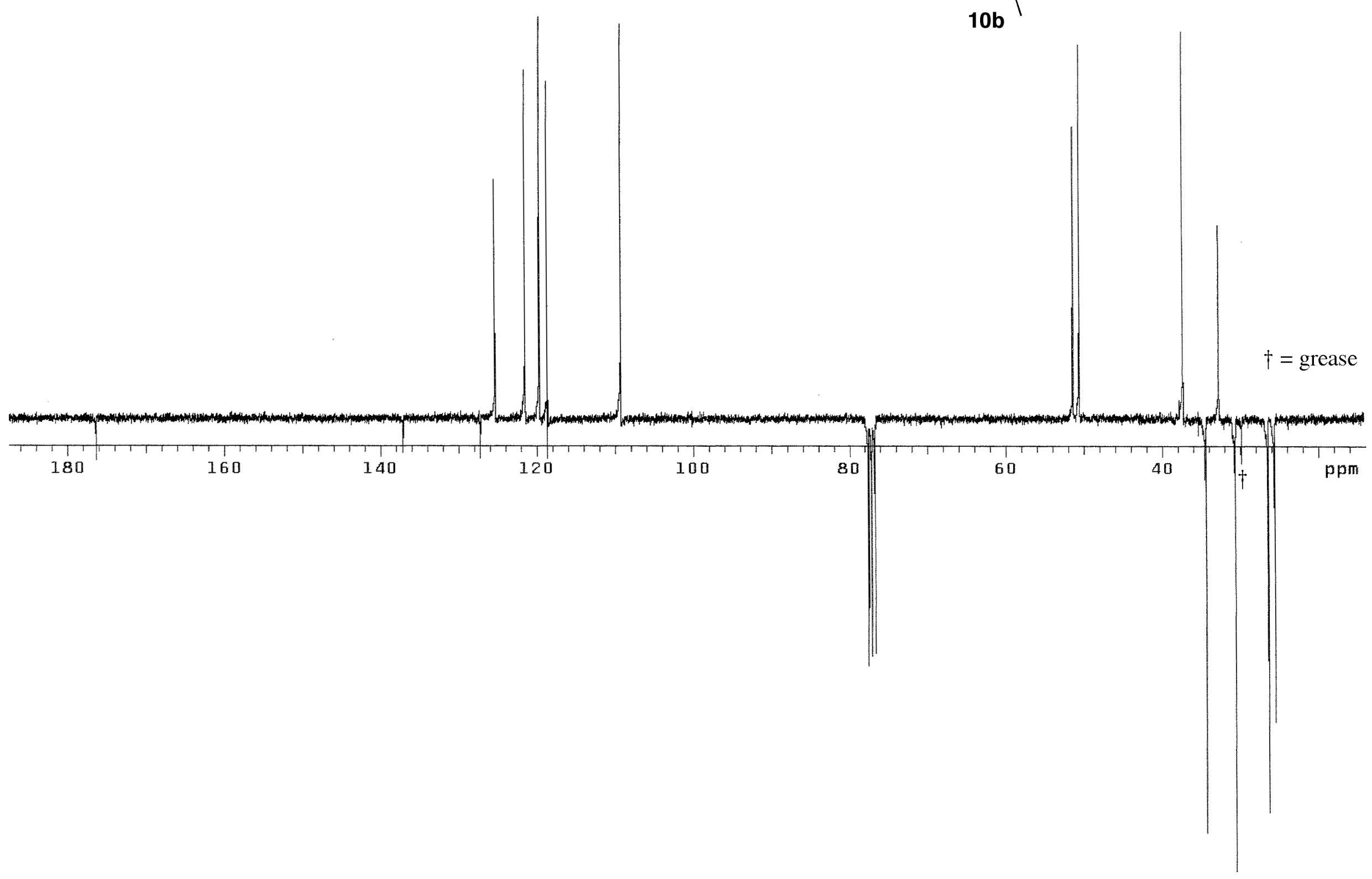

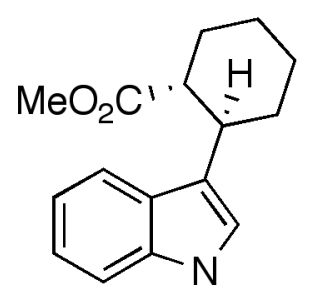

$10 \mathrm{~b}$ 
$75 \mathrm{MHz}$ APT ${ }^{13} \mathrm{C}$ NMR Spectrum of Compound 11a (recorded in $\mathrm{CDCl}_{3}$ )

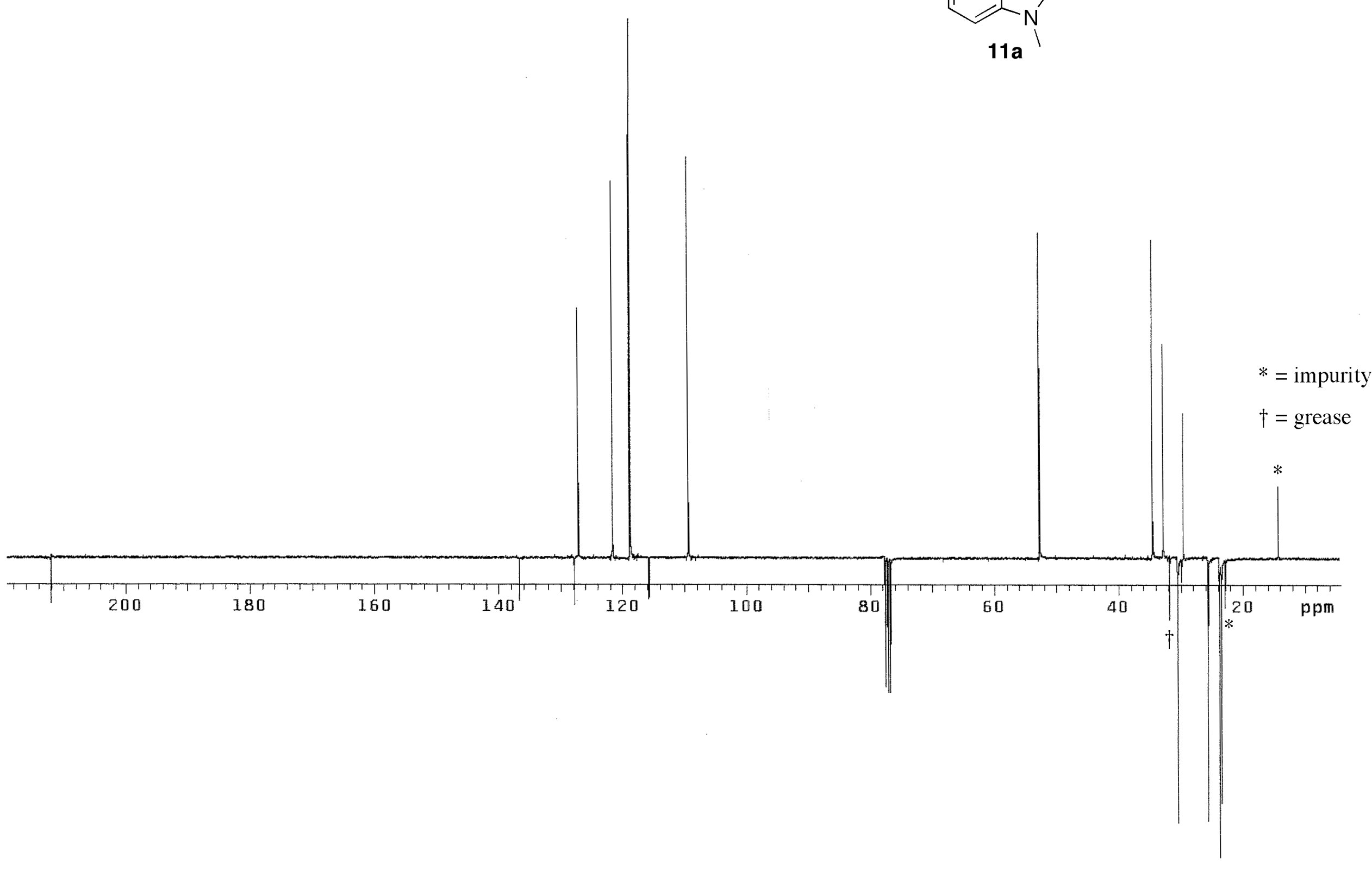


$75 \mathrm{MHz}$ APT ${ }^{13} \mathrm{C}$ NMR Spectrum of Compound $\mathbf{1 1 b}$ (recorded in $\mathrm{CDCl}_{3}$ )

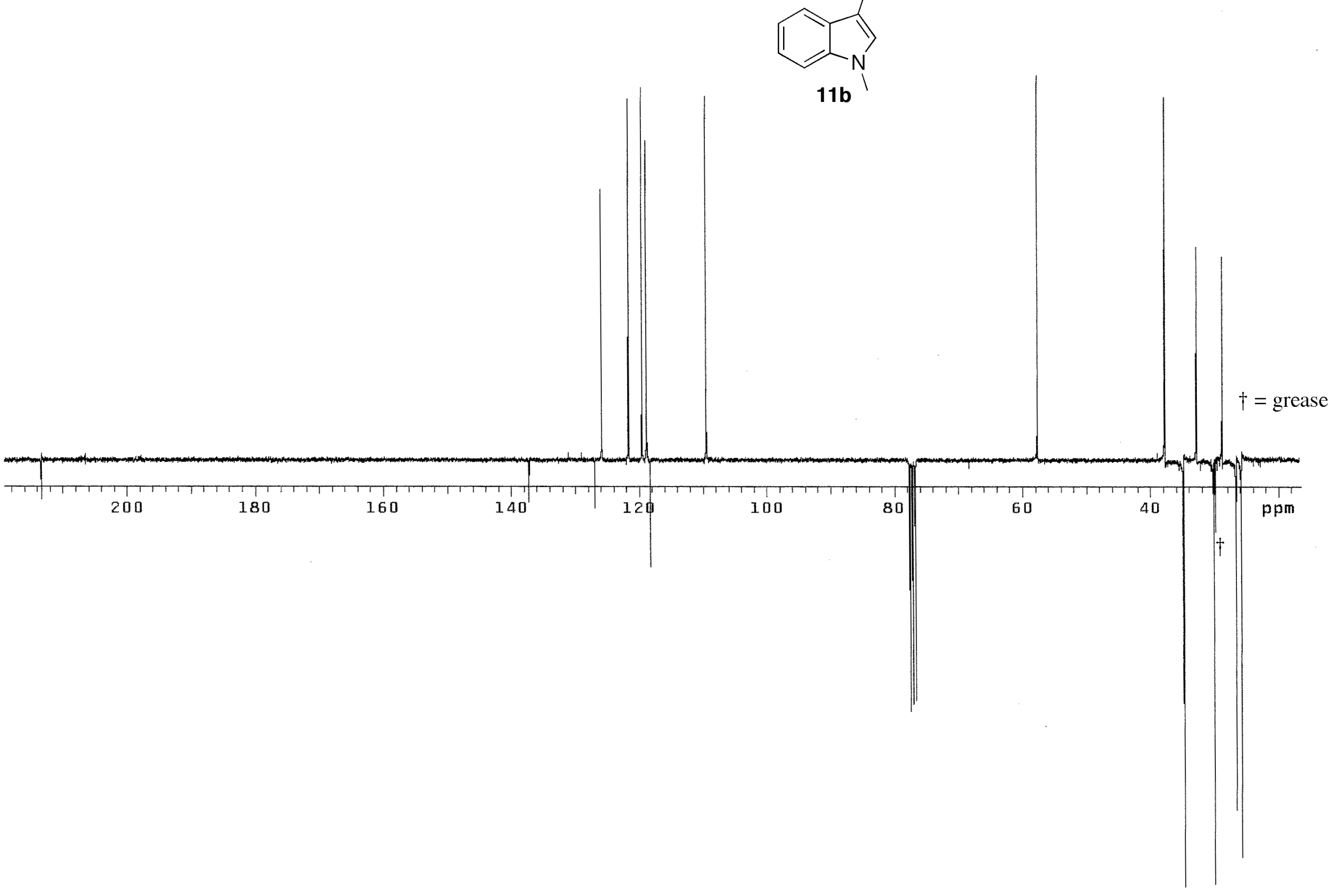


$300 \mathrm{MHz}{ }^{1} \mathrm{H}$ NMR Spectrum of Compound 12a

(recorded in $\mathrm{CDCl}_{3}$ )

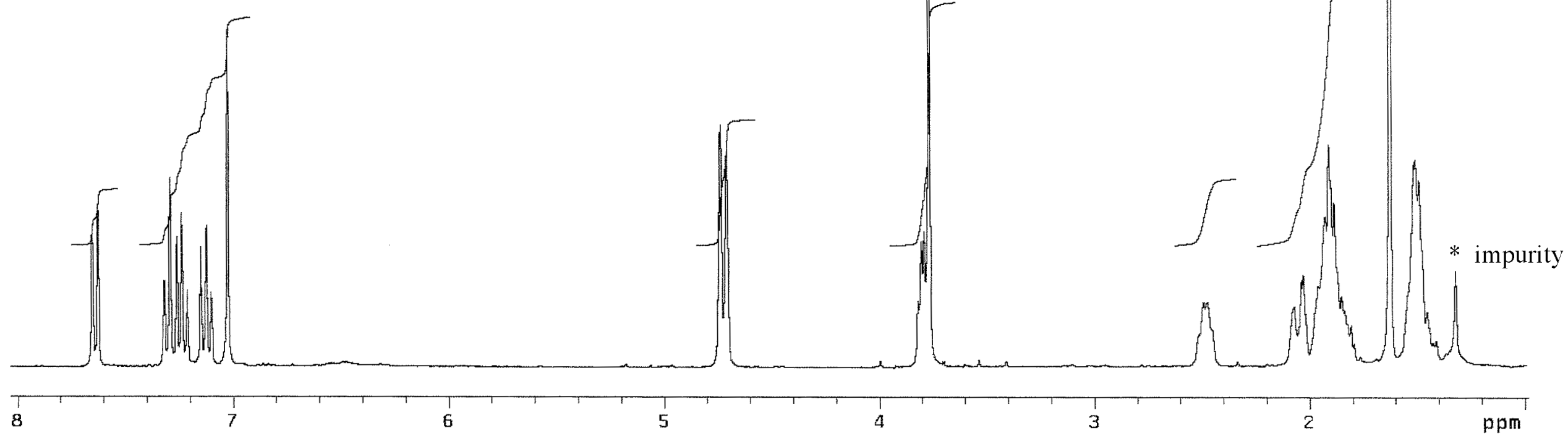


$300 \mathrm{MHz}{ }^{1} \mathrm{H}$ NMR Spectrum of Compound $\mathbf{1 2 b}$

(recorded in $\mathrm{CDCl}_{3}$ )

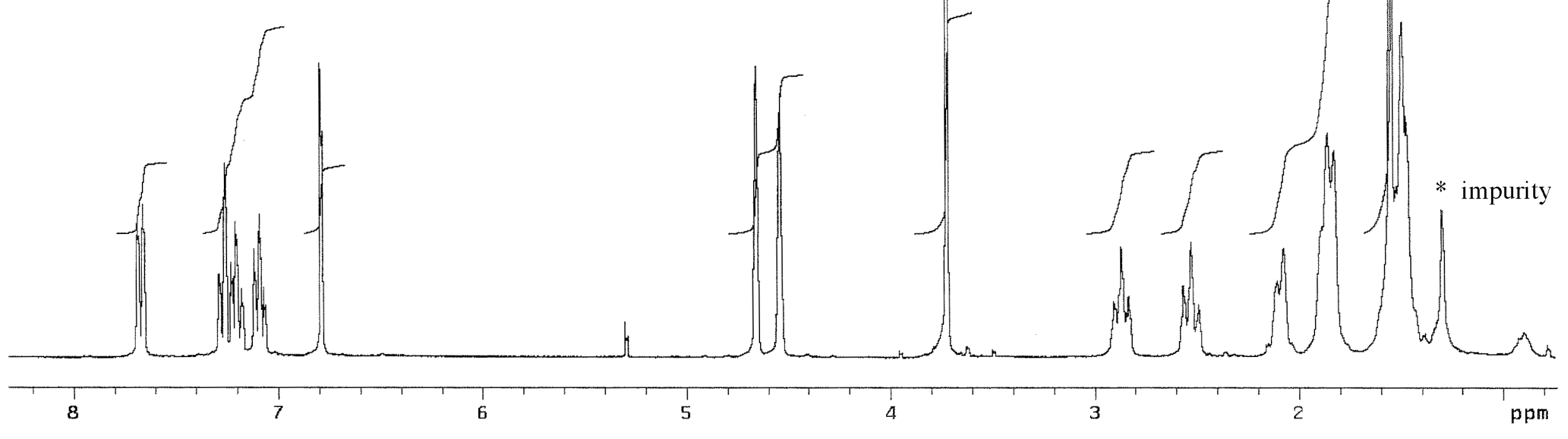


$75 \mathrm{MHz}$ APT ${ }^{13} \mathrm{C}$ NMR Spectrum of Compound 13a (recorded in $\mathrm{CDCl}_{3}$, signals at $\delta_{\mathrm{C}} 119.1,109.4,64.2,43.1$ $31.5,26.7,25.7$ and 25.0 due to the presence of minor amounts of isomer 13b)

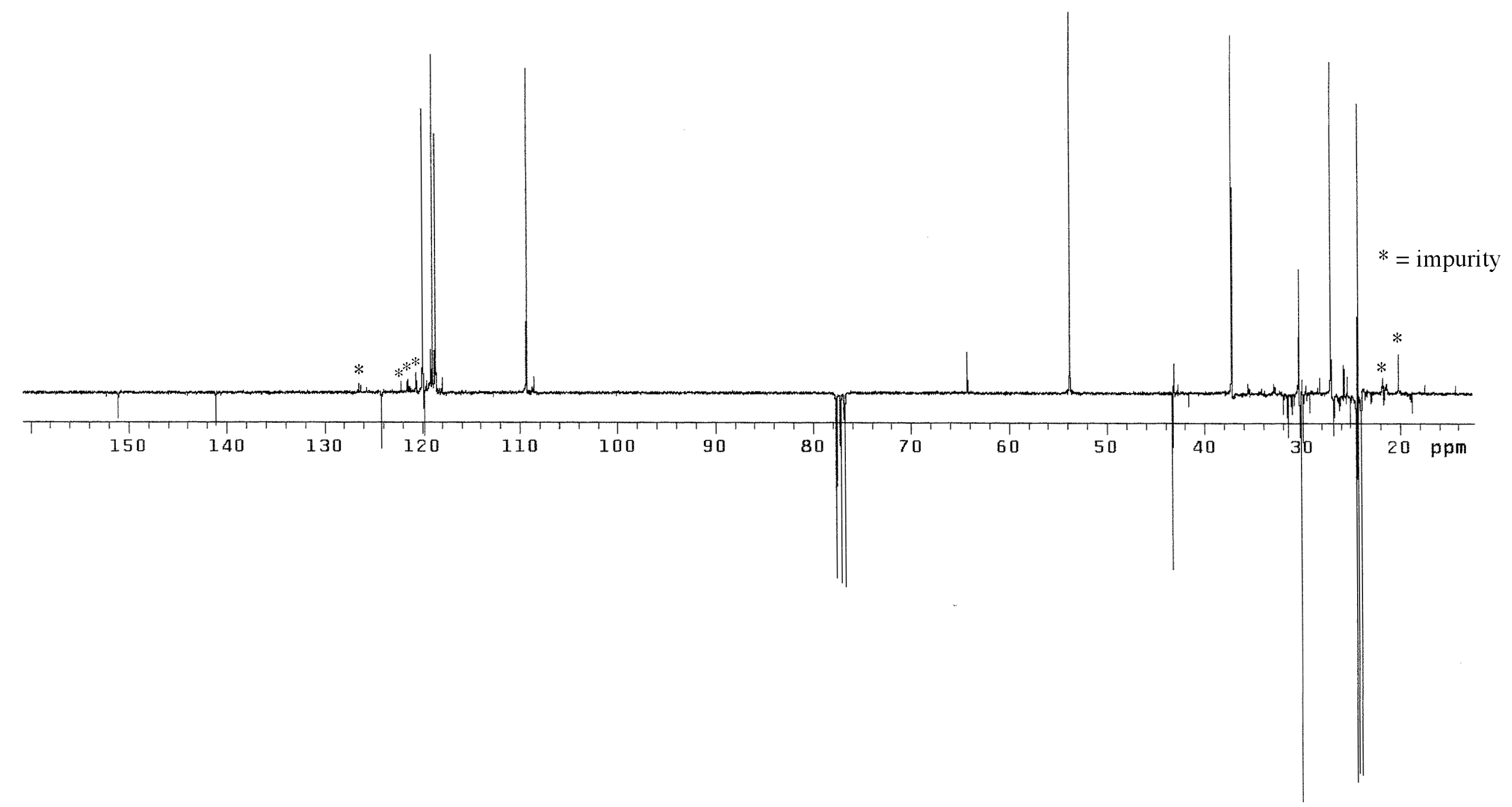

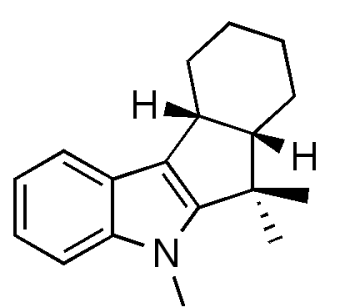

$13 a$ 
$75 \mathrm{MHz}$ APT ${ }^{13} \mathrm{C}$ NMR Spectrum of Compound 13b (recorded in $\mathrm{CDCl}_{3}$ )

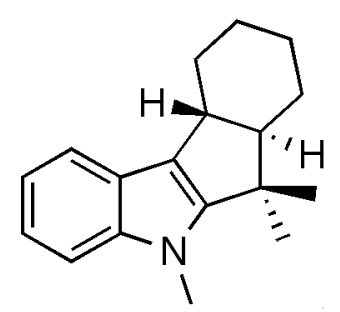

$13 b$

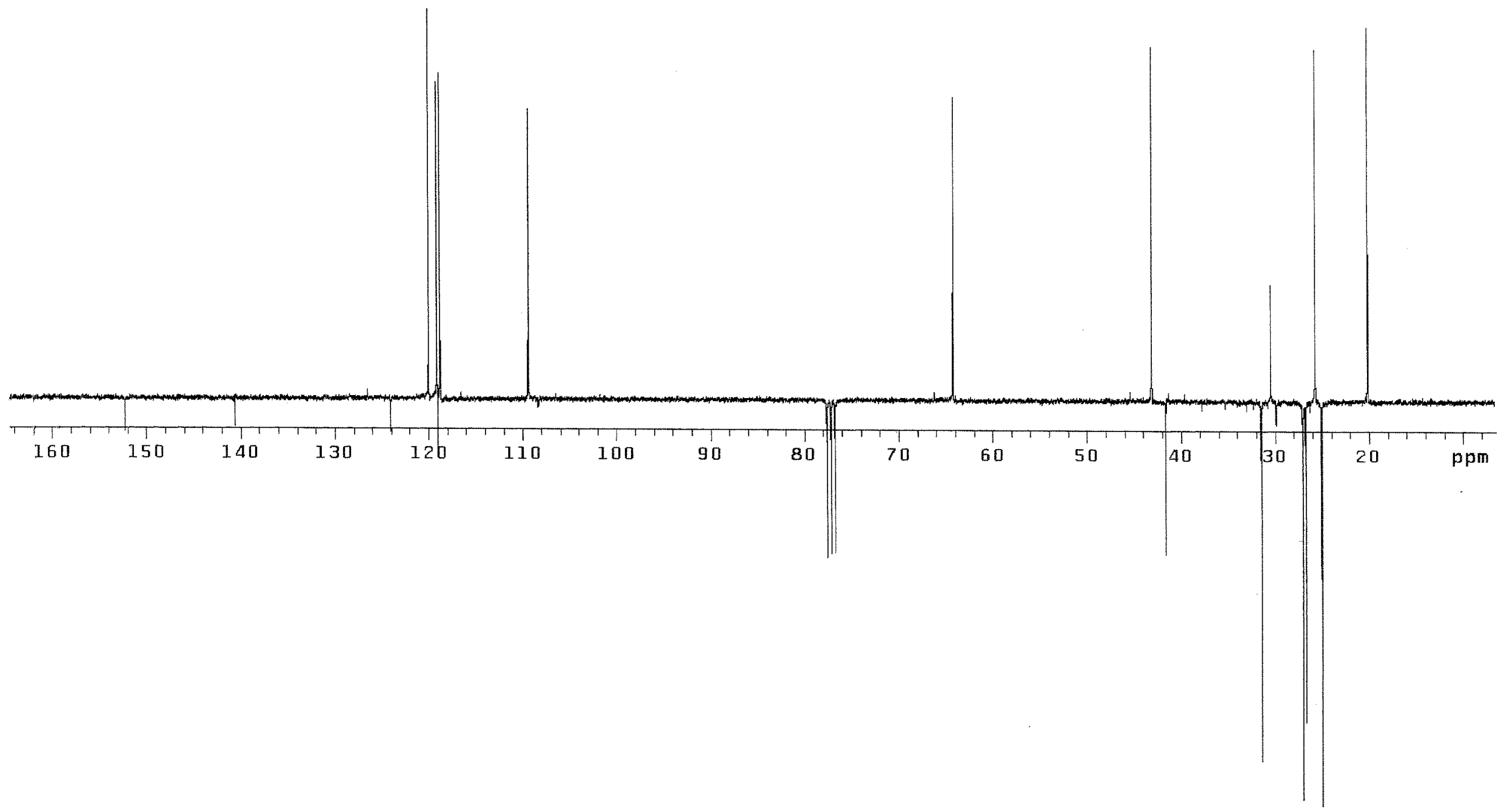


$300 \mathrm{MHz}{ }^{1} \mathrm{H}$ NMR Spectrum of Compound 17 (recorded in $\mathrm{CDCl}_{3}$ )
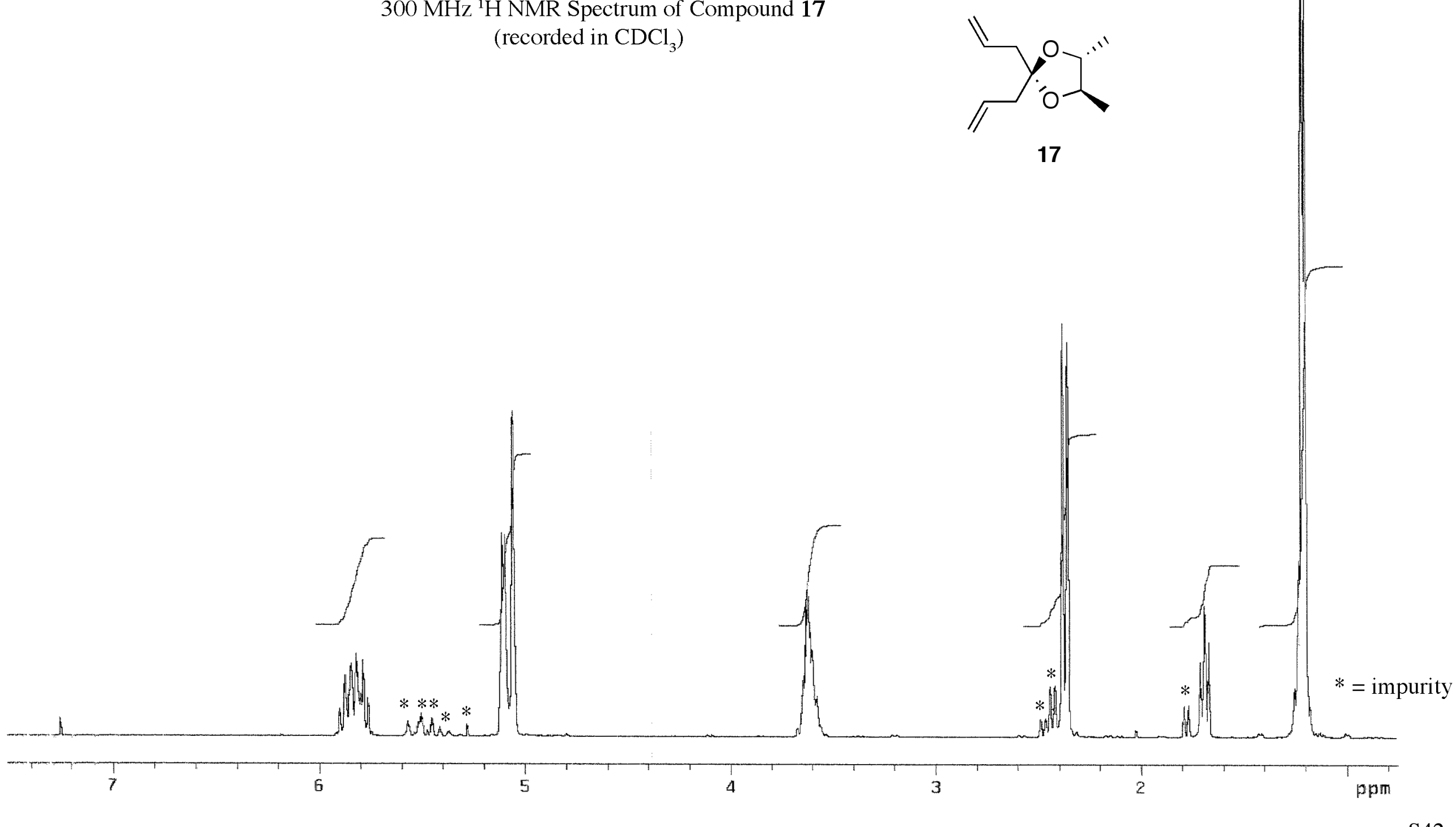


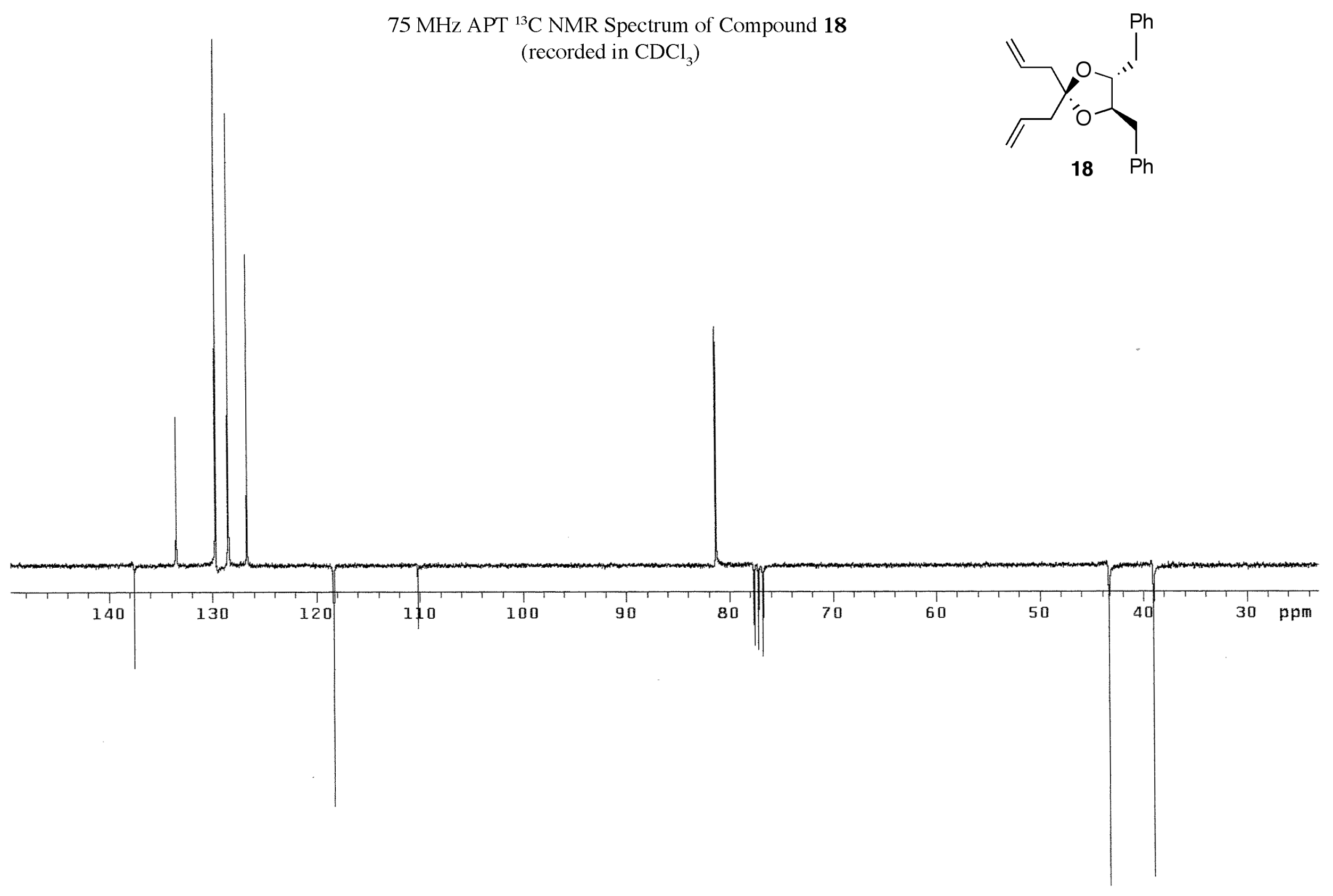


$75 \mathrm{MHz}$ APT ${ }^{13} \mathrm{C}$ NMR Spectrum of Compound 19 (recorded in $\mathrm{CDCl}_{3}$ )

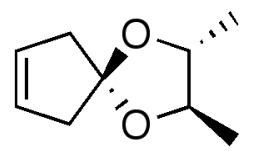

19

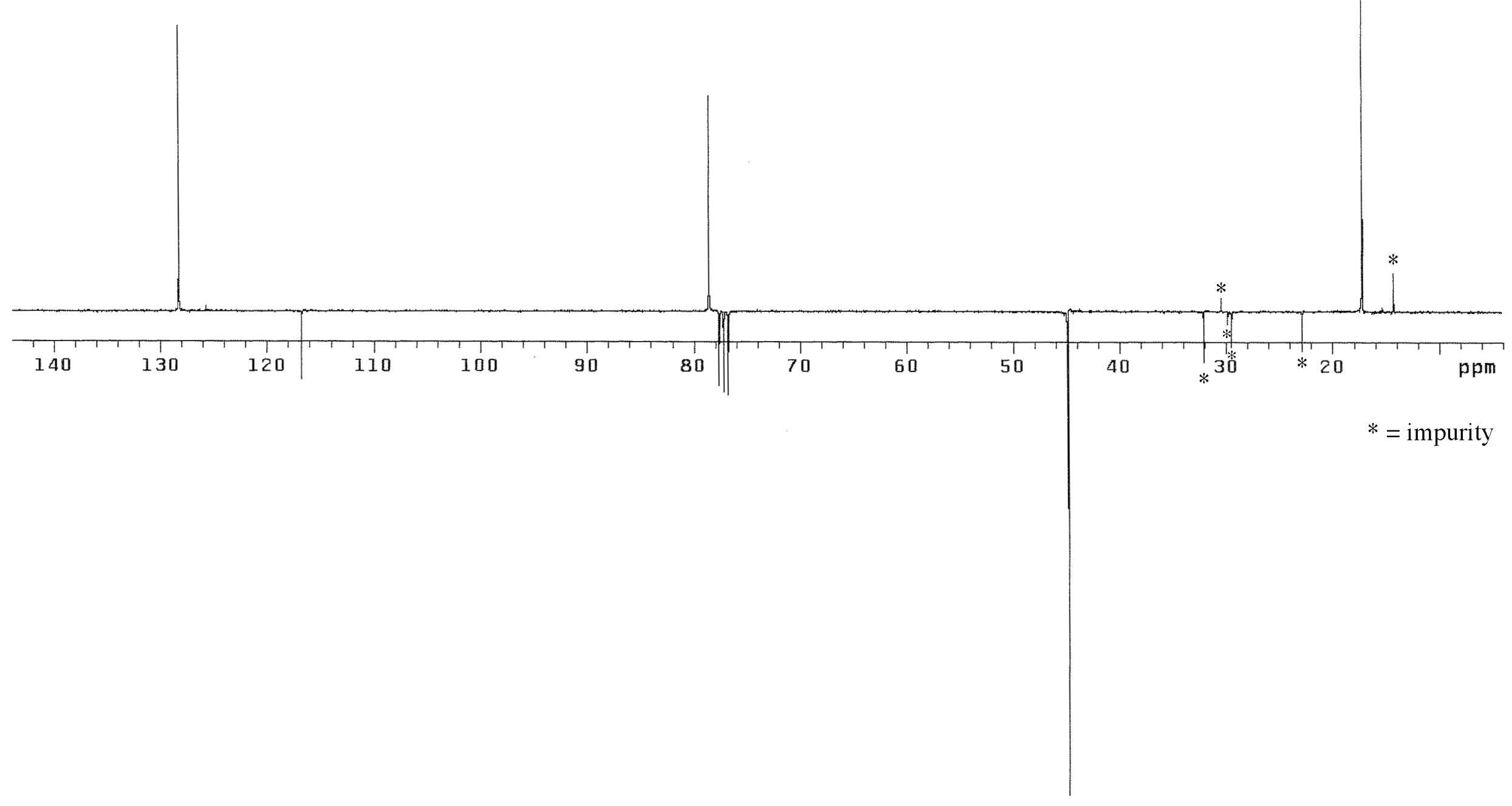


$75 \mathrm{MHz}$ APT ${ }^{13} \mathrm{C}$ NMR Spectrum of Compound 20 (recorded in $\mathrm{CDCl}_{3}$ )

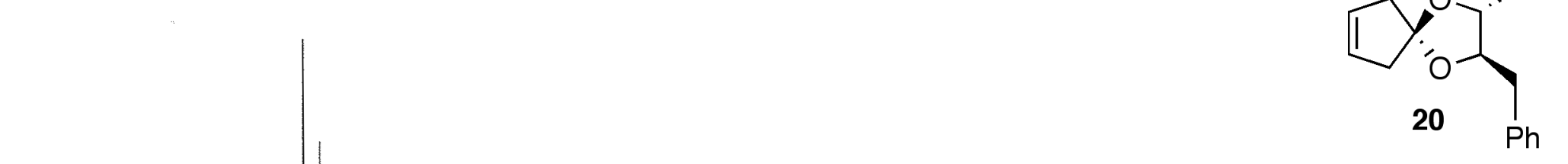


$75 \mathrm{MHz}$ APT ${ }^{13} \mathrm{C}$ NMR Spectrum of Compound 21 (recorded in $\mathrm{CDCl}_{3}$ )
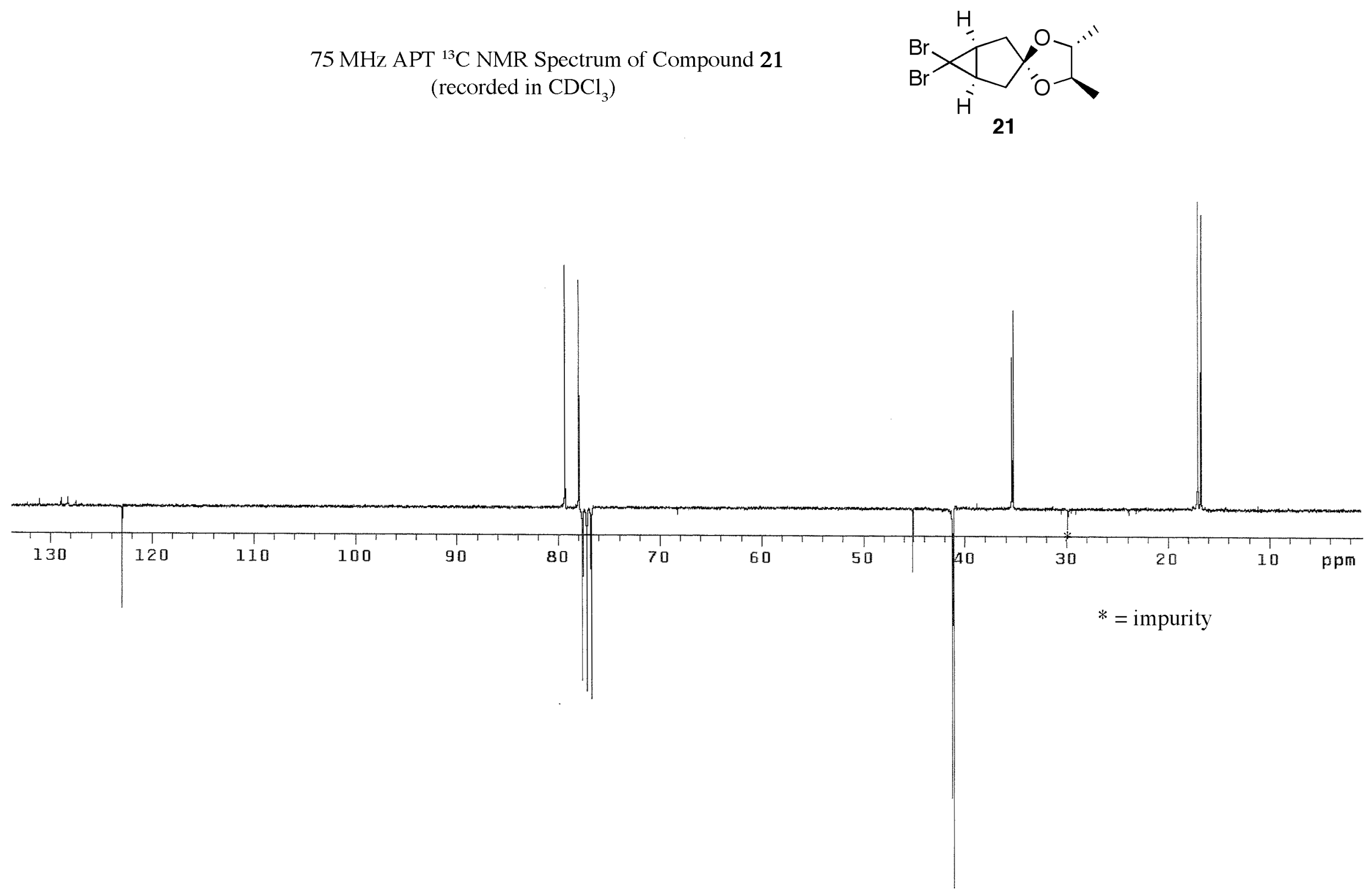
$75 \mathrm{MHz}$ APT ${ }^{13} \mathrm{C}$ NMR Spectrum of Compound 22 (recorded in $\mathrm{CDCl}_{3}$ )

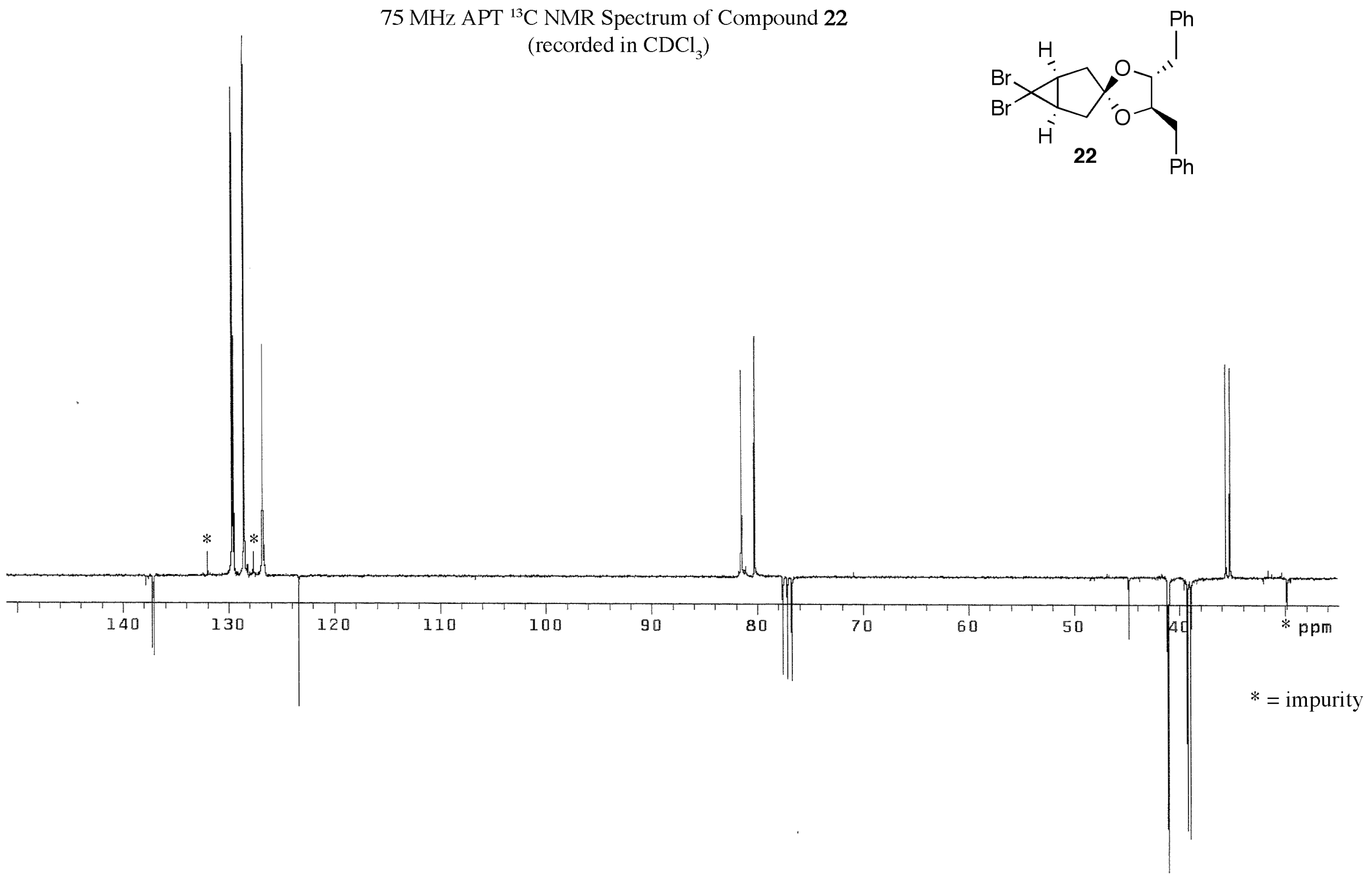


$300 \mathrm{MHz}{ }^{1} \mathrm{H}$ NMR Spectrum of Compound 23a

(recorded in $\mathrm{CDCl}_{3}$ )

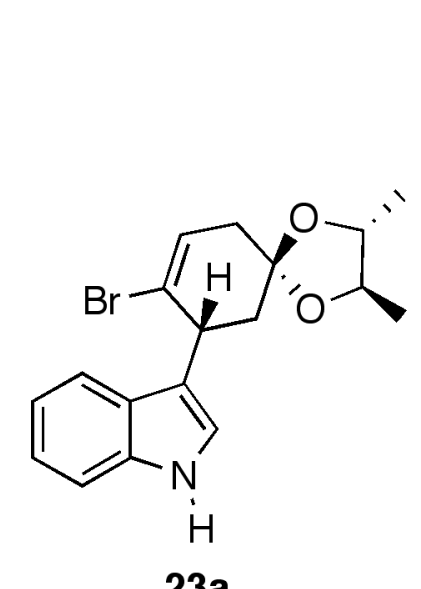

23a

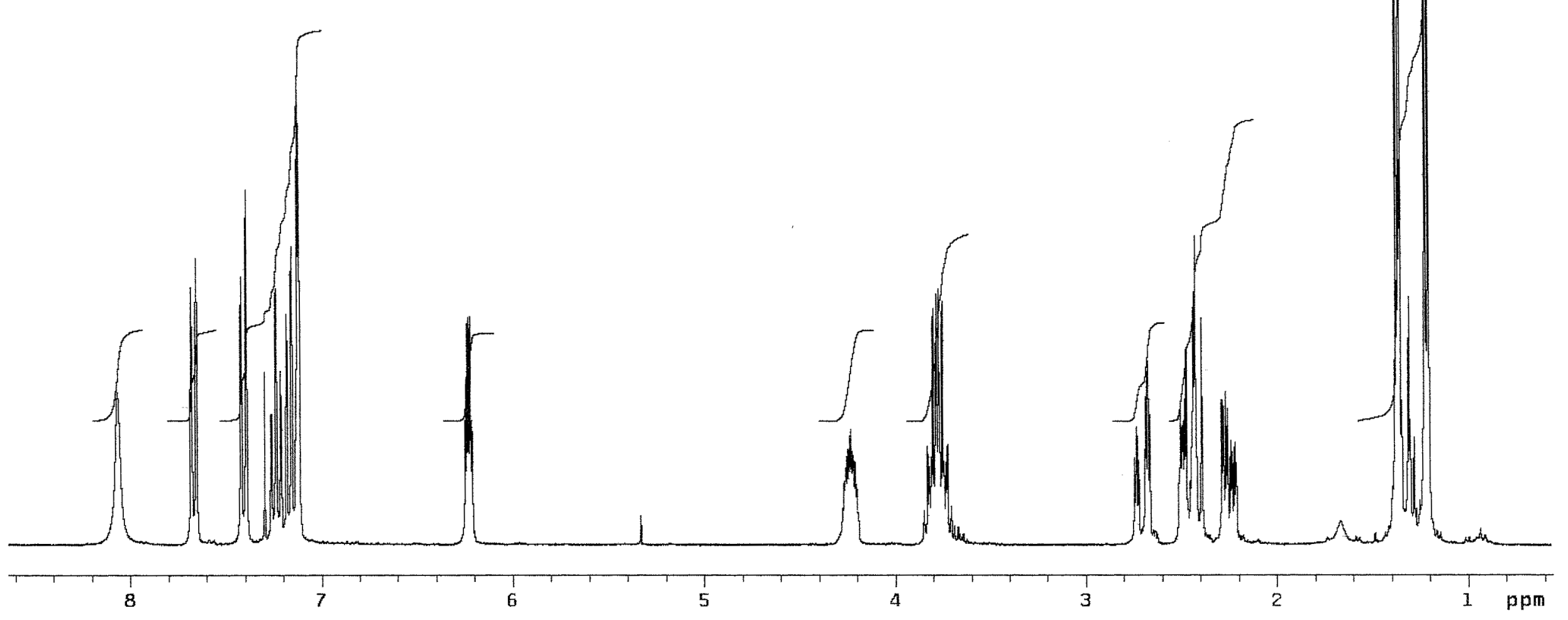


$300 \mathrm{MHz}{ }^{1} \mathrm{H}$ NMR Spectrum of Compound $23 \mathrm{~b}$

(recorded in $\mathrm{CDCl}_{3}$ )

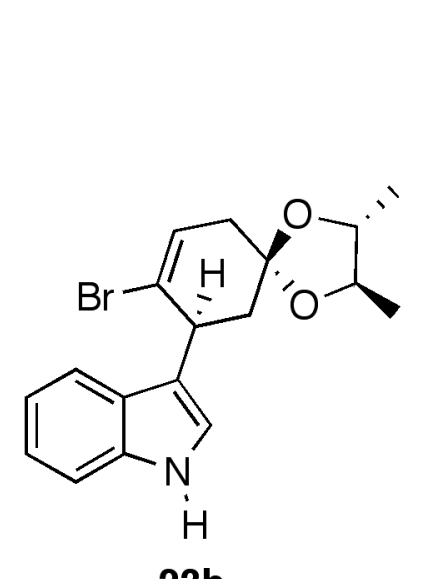

23b

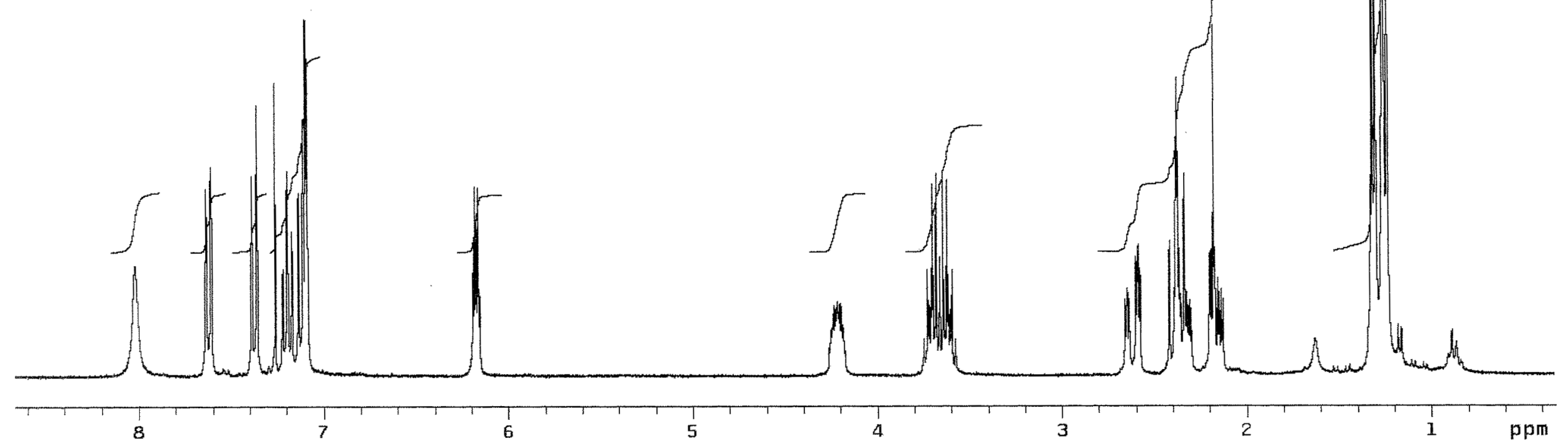


$75 \mathrm{MHz}$ APT ${ }^{13} \mathrm{C}$ NMR Spectrum of Compound 24a (recorded in $\mathrm{CDCl}_{3}$ )

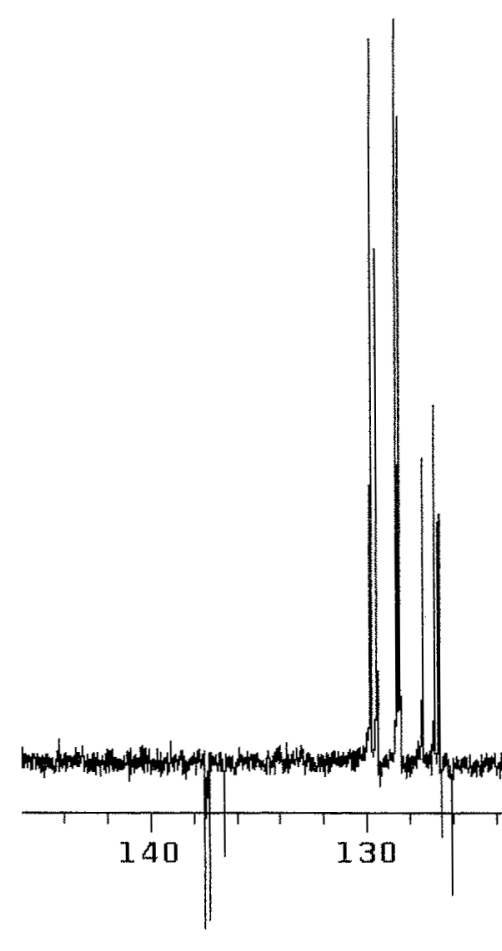

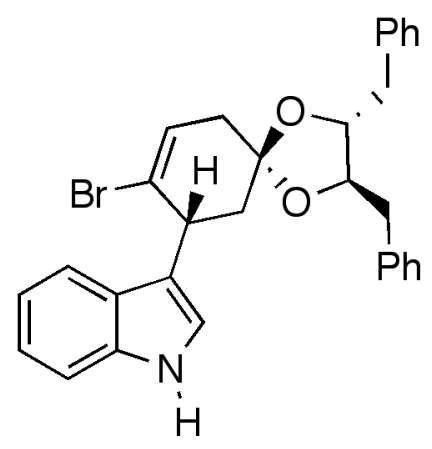

24a 
$75 \mathrm{MHz}$ APT ${ }^{13} \mathrm{C}$ NMR Spectrum of Compound 24b (recorded in $\mathrm{CDCl}_{3}$ )

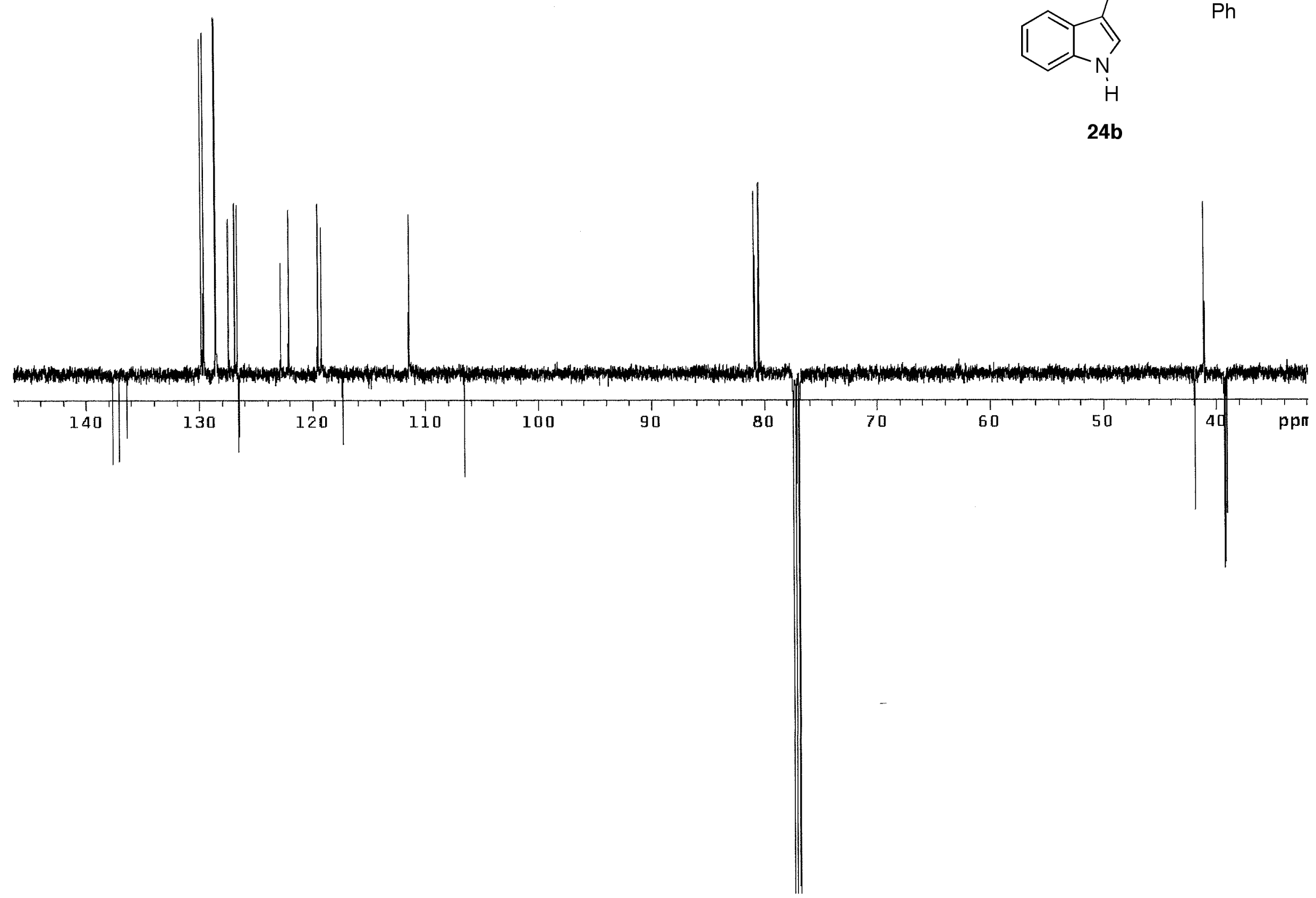

